\title{
UNIVERSITYOF BIRMINGHAM

Classifying shape of internal pores within AISi10Mg alloy manufactured by laser powder bed fusion using 3D X-ray micro computed tomography: Influence of processing parameters and heat treatment

\author{
Hastie, James C.; Kartal, Mehmet E.; Carter, Luke N.; Attallah, Moataz M.; Mulvihill, Daniel \\ M. \\ DOI: \\ 10.1016/j.matchar.2020.110225 \\ License: \\ Creative Commons: Attribution-NonCommercial-NoDerivs (CC BY-NC-ND)
}

Document Version

Peer reviewed version

Citation for published version (Harvard):

Hastie, JC, Kartal, ME, Carter, LN, Attallah, MM \& Mulvihill, DM 2020, 'Classifying shape of internal pores within AlSi10Mg alloy manufactured by laser powder bed fusion using 3D X-ray micro computed tomography: Influence of processing parameters and heat treatment', Materials Characterization, vol. 163, 110225.

https://doi.org/10.1016/j.matchar.2020.110225

Link to publication on Research at Birmingham portal

\footnotetext{
General rights

Unless a licence is specified above, all rights (including copyright and moral rights) in this document are retained by the authors and/or the copyright holders. The express permission of the copyright holder must be obtained for any use of this material other than for purposes permitted by law.

- Users may freely distribute the URL that is used to identify this publication.

- Users may download and/or print one copy of the publication from the University of Birmingham research portal for the purpose of private study or non-commercial research.

- User may use extracts from the document in line with the concept of 'fair dealing' under the Copyright, Designs and Patents Act 1988 (?)

- Users may not further distribute the material nor use it for the purposes of commercial gain.

Where a licence is displayed above, please note the terms and conditions of the licence govern your use of this document.

When citing, please reference the published version.
}

Take down policy

While the University of Birmingham exercises care and attention in making items available there are rare occasions when an item has been uploaded in error or has been deemed to be commercially or otherwise sensitive.

If you believe that this is the case for this document, please contact UBIRA@lists.bham.ac.uk providing details and we will remove access to the work immediately and investigate. 


\section{Journal Pre-proof}

Classifying shape of internal pores within AlSi10Mg alloy manufactured by laser powder bed fusion using 3D X-ray micro computed tomography: Influence of processing parameters and heat treatment

James C. Hastie, Mehmet E. Kartal, Luke N. Carter, Moataz M.

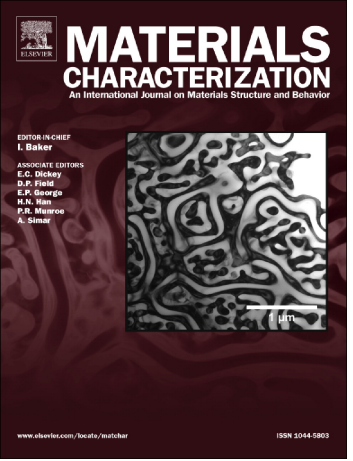
Attallah, Daniel M. Mulvihill

PII: S1044-5803(19)32971-7

DOI: https://doi.org/10.1016/j.matchar.2020.110225

Reference:

MTL 110225

To appear in:

Materials Characterization

Received date:

29 October 2019

Revised date:

25 January 2020

Accepted date: 24 February 2020

Please cite this article as: J.C. Hastie, M.E. Kartal, L.N. Carter, et al., Classifying shape of internal pores within AlSi10Mg alloy manufactured by laser powder bed fusion using 3D X-ray micro computed tomography: Influence of processing parameters and heat treatment, Materials Characterization (2020), https://doi.org/10.1016/ j.matchar.2020.110225

This is a PDF file of an article that has undergone enhancements after acceptance, such as the addition of a cover page and metadata, and formatting for readability, but it is not yet the definitive version of record. This version will undergo additional copyediting, typesetting and review before it is published in its final form, but we are providing this version to give early visibility of the article. Please note that, during the production process, errors may be discovered which could affect the content, and all legal disclaimers that apply to the journal pertain.

C) 2020 Published by Elsevier. 
Classifying shape of internal pores within AlSi10Mg alloy manufactured by laser powder bed fusion using 3D X-ray micro computed tomography: influence of processing parameters and heat treatment

James C. Hastie ${ }^{\mathrm{a} *}$, Mehmet E. Kartal ${ }^{\mathrm{a}}$, Luke N. Carter ${ }^{\mathrm{b}}$, Moataz. M. Attallah ${ }^{\mathrm{c}}$, Daniel M. Mulvihill ${ }^{\mathrm{d}}$

a - School of Engineering, University of Aberdeen, UK

b - School of Chemical Engineering, University of Birmingham, UK

c - School of Metallurgy and Materials, University of Birmingham, UK

d - School of Engineering, University of Glasgow, UK

*_Corresponding author. Email: r03jh15@abdn.ac.uk 


\title{
Classifying shape of internal pores within AISi10Mg alloy manufactured by laser powder bed fusion using 3D X-ray micro computed tomography: influence of processing parameters and heat treatment
}

Keywords: Laser powder bed fusion; selective laser melting; X-ray micro computed tomography; porosity classification; hot isostatic pressing; T6 heat treatment

\begin{abstract}
Internal porosity of metallic parts manufactured by laser powder bed fusion (LPBF) is governed by processing parameters including laser power, scanning speed, scan spacing and layer thickness. To fully understand the influence of processing parameters it is important to categorise the shape of process defects (pores) in 3D beyond the degree of sphericity alone. In the present paper, AlSi10Mg samples were manufactured using 30 unique LPBF parameter combinations and analysed using high resolution X-ray micro computed tomography $(\mathrm{X} \mu \mathrm{CT})$. The shapes of individual pores are classified and studied using an approach based on the similarity of 3D pore descriptors with simplified artificial objects. Porosity within high as-fabricated densification builds can be reduced to virtually negligible by hot isostatic pressing (HIPping), which was found to fully or partially close (flatten) pores. Subsequent T6 treatment causes pores to reopen and resemble their original shape. The effects of treatment are sensitive to pore size.
\end{abstract}

\section{Introduction}

Additive manufacturing (AM) affords the ability to produce complex final-shape components directly from raw material powder, thereby eliminating the need for subtractive manufacturing stages such as machining of cast stock. The advent of widespread AM production has the potential to drastically streamline metallic part manufacturing by decreases in: steps from raw material to final part; material wastage; environmental burden and $\mathrm{CO}_{2}$ footprint; and manufacturing costs, with a focus on low volume production and customer flexibility.

Laser powder bed fusion (LPBF), also broadly referred to as 'selective laser melting (SLM)', is an AM process involving layer by layer melting of deposited metallic powder by computer-controlled laser to build a desired component. Densification of the final part is governed by feedstock (powdered) material properties, LPBF processing parameters (i.e. laser power, scanning speed etc.) and scan strategy. Aluminium alloys including traditionally cast AlSi10Mg used extensively for lightweight aerospace and automotive components are widely considered as LPBF candidates. However, effective laser processing of aluminium can be especially challenging due to susceptibility to oxidation, high reflectivity (necessitating high laser powers for melting) and high thermal conductivity causing rapid dissipation of heat away from the scanned area [1]. At present, AlSi10Mg components produced by LPBF have largely been limited to non-critical applications due to a lack of understanding of mechanical behaviour and microstructural quality.

Existing research has focused extensively on optimising LPBF processing parameters, powder properties and scanning strategies for producing high densification AlSi10Mg parts and enhancing mechanical performance by conventional aluminium heat treatments. In general, the majority of studies have been limited to the use of optical microscopy (OM) and scanning electron microscopy (SEM) to evaluate microstructure and ascertain free-surface porosity volume in 2D [2-12]. However, this does not provide information on the subsurface where pore morphology may differ, particularly when one considers there are practical limits to the amount of internal slices that can be exposed, polished and scanned per specimen. A clear trend in the use of $\mathrm{X}$-ray micro computed tomography $(\mathrm{X} \mu \mathrm{CT})$ as a robust non-destructive means of 
examining and quantifying 3D pore characteristics, including size, shape, frequency and location, is evident in the latest state-of-the-art [13-19]. X $\mu \mathrm{CT}$ offers two main advantages over conventional cross-sectioning and imaging techniques [15]: (i) the specimen is not subjected to grinding and polishing that may alter pore morphology on the free-surface; (ii) a fully invasive 3D image of the material is generated instead of a limited number of 2D cross-sections, revealing significantly more and drastically reducing manual requirements. In reality, the relatively large size of full-scale functional LPBF-fabricated parts will severely limit the highest possible resolution of $\mathrm{X} \mu \mathrm{CT}$ for given limited scanning time and pores of significant size will unavoidably be missed. Therefore, analysing the porous structure of small-scale specimens is an important step in understanding LPBF processing of AlSi10Mg, albeit one must exercise caution when interpreting small-scale knowledge for eventual full-scale applications.

In $\mathrm{X} \mu \mathrm{CT}$ analysis of LPBF-fabricated parts the size of individual pores has most often been measured using equivalent diameter, that is, the diameter of a sphere of volume equivalent to the pore $\left(V_{\text {pore }}\right)$ determined using 3D image thresholding software, expressed as:

$$
d_{\mathrm{eq}}=\sqrt[3]{\frac{6 V_{\mathrm{pore}}}{\pi}}
$$

Pore shape is frequently classified using the basic geometric equation for sphericity i.e. the ratio of surface area of an equivalent volume sphere to surface area of the pore $\left(A_{\text {pore }}\right)$ :

$$
\Psi=\frac{\pi^{1 / 3}\left(6 V_{\text {pore }}\right)^{2 / 3}}{A_{\text {pore }}}
$$

where $\Psi=1.0$ corresponds to a perfect sphere and low $\Psi$ to non-spherical (irregular) shape. The degree of sphericity has been widely used as a means of classifying defect type and identifying formation mechanisms in LPBF-fabricated parts. Spherical pores are indicative of residual gases in the powder, or gases that become trapped due to melt flow turbulence when processing with excessive laser energy or high scanning speeds, and are typically isolated within solidified melt pools $[3,4,17,20]$. High energy may also cause localised vaporisation leading to the formation of spherical pores deep in solidified pools $[4,5]$. This is known as keyhole porosity, which can manifest as larger spherical or irregular shapes with keyhole instability (rapid solidification with incomplete filling of gaps) [2-5]. Irregular pores are most commonly attributed to the lack of fusion between adjacent layers or partially melted powder particles when applying insufficient energy [4,20]. Failure to effectively disrupt oxide films that are present in the powder and prevent consolidation is also a known mechanism for irregular defect formation in low energy processing of Al alloys $[1,5,8,16,17,21]$. Oxide film defects can be introduced by overheating with high energy or by melt pool instability (e.g. driven by Marangoni force and recoil pressure [22]) at high scanning speeds.

Whilst sphericity combined with location can be used to distinguish between gas-type pores and other specific defect mechanisms, it cannot be used to adequately quantify the 3D shape of highly irregular pores alone or when used in conjunction with a single longest-to-shortest dimension aspect ratio. For example, features exhibiting low sphericity may be relatively equidimensional or conversely elongated along one or two measures in 3D, e.g. long and axisymmetric or flat and plate-like. Different physical pore shapes may influence macroscopic mechanical properties and part performance to differing extents and as such, to further understand the influence of key processing parameters and heat treatments it is necessary to adopt novel approaches for classifying pore shape beyond the degree of roundness. 
In the present paper, AlSi10Mg samples were manufactured using 30 unique combinations of LPBF processing parameters yielding a range of very low to high densifications. Internal porosity was analysed by $\mathrm{X} \mu \mathrm{CT}$ scanning with resolution superior to the majority of preceding AlSi10Mg studies as per the authors' best knowledge. The influence of key processing parameters on pore shape was investigated using an approach to categorise individual pores based on similarity of 3D pore descriptors to simplified geometric objects. Several samples were subjected to hot isostatic pressing (HIPping) followed by T6 (solutionising and artificial ageing) and rescanned after each stage to evaluate changes to porosity.

\section{Materials and Methods}

\subsection{Laser Processing and Heat Treatment Parameters}

AlSi10Mg samples were fabricated using a Concept Laser M2 Cusing LPBF system. Composition of the alloy powder (size range $15-53 \mu \mathrm{m}$ ) supplied by LPW Technology is given in Table 1. The powder has a reasonable flowability and Hausner ratio for LPBF despite non-spherical morphology and is similar to the powder used by Read et al. [5]. Samples were built in an argon environment with oxygen content controlled down to 100ppm. A total of $3412 \mathrm{~mm}$-long cylindrical samples with $3.5 \mathrm{~mm}$ diameter were manufactured covering 30 combinations of laser power $(150,250,350 \mathrm{~W})$, scanning speed $(500,1500,2500 \mathrm{~mm} / \mathrm{s})$, scan spacing $(45,75,105 \mu \mathrm{m})$ and layer thickness $(30,60 \mu \mathrm{m})$. These four processing parameters are the most influential on densification, microstructure and mechanical properties [21,23]. In this study, parameters and conditions kept constant, such as sample size and geometry, platform conditions, build direction and scanning strategy, were not varied as parameters for investigation purely due to a limited number of samples and $\mathrm{X} \mu \mathrm{CT}$ time, however the approach outlined in this paper could equally be used to study their respective influence on porosity. Samples were built vertically along the cylindrical axis ( $Z$ direction) and scanned with $150 \mu \mathrm{m}$ laser track width by a raster strategy in the transverse $X Y$ plane. Two sets of three samples were produced using identical parameters to validate manufacturing and post-processing repeatability. Sample parameters are summarised in Appendix A. Based on the parameters chosen for investigation in this work, the volumetric energy density $(V E D)$ function $\left(\mathrm{J} / \mathrm{mm}^{3}\right)$ is a simple indicator of how much energy is delivered to the material, expressed as:

$$
V E D=\frac{P}{v \cdot h \cdot t}
$$

where $P, v, h$ and $t$ are laser power $(\mathrm{W})$, scanning speed $(\mathrm{mm} / \mathrm{s})$, scan spacing $(\mathrm{mm})$ and layer thickness $(\mathrm{mm})$ respectively.

Table 1. AlSi10Mg composition (Wt. \%)

\begin{tabular}{|c|c|c|c|c|c|c|c|c|c|}
\hline Al & $\mathbf{S i}$ & $\mathbf{M g}$ & $\mathbf{F e}$ & $\mathbf{N i}$ & $\mathbf{Z n}$ & $\mathbf{T i}$ & Mn & $\mathbf{P b}$ & Sn \\
\hline Bal. & 9.92 & 0.291 & 0.137 & 0.04 & 0.01 & 0.006 & 0.004 & 0.004 & 0.003 \\
\hline
\end{tabular}

Six samples were subjected to HIPping and T6 procedures in accordance with the standard AlSi10Mg material as outlined in Table 2. The time between T6 heating stages was < $1 \mathrm{hr}$. Samples were selected for treatment based on the as-built results presented later in Section 3.1. 
Table 2. Heat treatment parameters

\begin{tabular}{ll}
\hline HIPping & T6 \\
\hline $2 \mathrm{hrs} @ 500^{\circ} \mathrm{C} / 100 \mathrm{MPa}$ & $5 \mathrm{hrs} @ 530^{\circ} \mathrm{C}$ \\
$5^{\circ} \mathrm{C} /$ min heating/cooling & Water quench \\
& $8 \mathrm{hrs} @ 160^{\circ} \mathrm{C}$ \\
& Air cooling \\
\hline
\end{tabular}

\subsection{Post-processing}

\subsubsection{X-ray Micro Computed Tomography}

$\mathrm{X} \mu \mathrm{CT}$ was conducted using the ZEISS VersaXRM-410, which has a minimum spatial resolution of $0.9 \mu \mathrm{m}$, minimum voxel size of $0.1 \mu \mathrm{m}$, maximum power output of $10 \mathrm{~W}$ and maximum voltage of $150 \mathrm{kV}$, and can be fitted with a micro tension/compression test rig for in-situ X $\mu \mathrm{CT}$ scanning at incremental stages of loading (e.g. see $[24,25]$ ). Samples were scanned using $80 \mathrm{kV}$ X-ray beam energy and $10 \mathrm{~W}$ power. A magnification of $4 \mathrm{X}$ was used to achieve $1024 \mathrm{X} 1024$ pixel (px) resolution with $2.96 \mu \mathrm{m}$ pixel size and $3 \mathrm{~mm}^{3}$ field of view. Exposure time was set at $6 \mathrm{~s}$, giving intensity values $>5000$ for optimal signal-to-noise ratio. Sets of 3200 projections were captured over $360^{\circ}$ sample rotation. Xradia XMReconstructor software was used to reconstruct $2 \mathrm{D}$ radiographs into $3 \mathrm{D}$ and beam hardening and centre shift artefacts were removed. To the authors' best knowledge the $\mathrm{X} \mu \mathrm{CT}$ specifications outlined here are superior to the majority of preceding

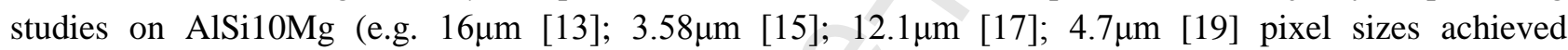
previously) particularly given the large sample set (34 total) and trade-off between image quality and scanning time.

\subsubsection{Optical Microscopy}

Some inherent uncertainty associated with the method and implementation of X $\mu \mathrm{CT}$ post-processing can be expected. Therefore, seven random samples were scanned in as-built condition using OM as a means of validating repeatability using an entirely separate method. Samples were cut through the centre leaving two separate sections ( $400 \mu \mathrm{m}$ blade thickness) and mounted in resin for mechanical polishing: five minutes with a P220 resin bonded diamond disc; ten minutes with $9 \mu \mathrm{m}$ diamond suspension; 45 minutes with $0.04 \mu \mathrm{m}$ colloidal silica suspension. Polishing was carried out at 150rpm. Sectioned surfaces were scanned using Alicona InfiniteFocus G4 (20X objective) and Thorlabs' Cerna (10X objective) microscopes and porosities were averaged from the two. InfiniteFocus and Cerna pixel sizes were 0.44 and $0.55 \mu \mathrm{m}$ respectively.

\subsubsection{Segmentation Analysis}

The software Avizo 9 was used to evaluate porosity volume and individual pore size/shape characteristics by thresholding of $\mathrm{X} \mu \mathrm{CT}$ images. A discrete 600px cubic sub-volume was analysed within each sample image at identical middle location to avoid edge effects and ensure consistent comparison between samples. A nonlocal means filter was applied and features smaller than $1 \mathrm{px}$ were neglected. The built-in thresholding tool available within the segmentation editor was employed. As with any analysis based on a region of interest, results are sensitive to size and location of the region to some extent. To assess repeatability a second X $\mu \mathrm{CT}$ porosity volume was obtained using a smaller 500px sub-volume at random locations within the seven samples also checked by OM. Porosity of OM images was analysed using the default thresholding method (a variation of the iterative method in [26]) on the software ImageJ. Analysis was performed on a central square equivalent to the $600 \times 600 \mathrm{px} \mathrm{X} \mu \mathrm{CT}$ section and features smaller than $1 \mathrm{px}$ were neglected. 


\subsubsection{Pore Shape Classification}

The sphericity ratio has been the most commonly used means of quantifying individual pore shape within LPBF-fabricated AlSi10Mg and attributing formation mechanism in studies utilising X $\mu \mathrm{CT}$ (e.g. [17-19]). In this study, pores analysed by $\mathrm{X} \mu \mathrm{CT}$ are classified in 3D using an approach based on simplified artificial objects as employed elsewhere for alternative porous media [27-29]. To quantify shape we firstly acquire three pore descriptors, $L, l$ and $S$, where $L$ is the longest pore dimension, $l$ is the longest dimension perpendicular to $L$ and $S$ is the smaller dimension perpendicular to both $L$ and $l$. The descriptors are determined by two methods. In the bounding box (BB) method, pore dimensions are taken along global axes $X, Y$ and $Z$, i.e. the pore is bounded by the 3D space defined by $D x, D y$ and $D z$, where the descriptors $L, l$ and $S$ are the largest, middle and smallest of the three respectively. In the Feret caliper (FC) method, a maximum length $(L)$, corresponding minimum width $(S)$ in the same plane and maximum diameter perpendicular to $S$ ( $l$ ) are determined for a number of orientations. Here, measurements for each pore were taken in 100 directions on Avizo to determine maximum Feret length and minimum width. Figure 1 shows the 3D dimensions used to describe a pore using BB and FC methods.

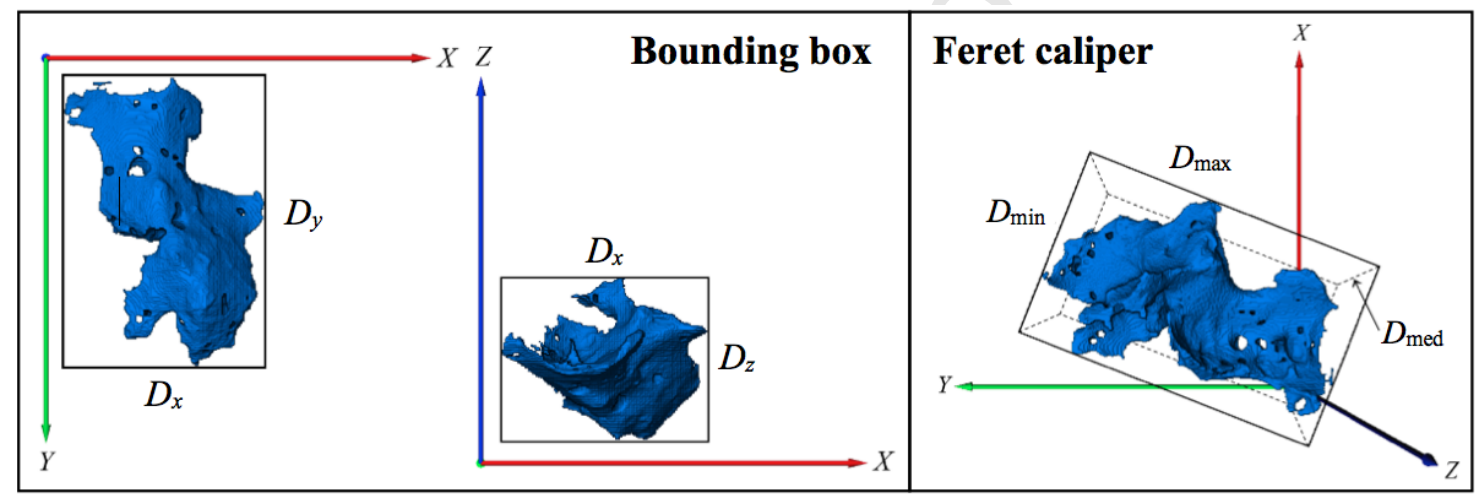

Figure 1. Descriptors used in bounding box and Feret caliper methods, where $L, l$ and $S$ correspond respectively to the largest, middle and smallest bounding box dimensions ( $D_{y}, D_{x}, D_{z}$ as shown) or Feret diameters $\left(D_{\max }, D_{\text {med }}, D_{\min }\right)$ of an individual pore

Using $S / l$ and $l / L$ ratios, pores are classified according to the closeness of their $3 \mathrm{D}$ space to simplified geometric objects as per Figure 2: rod-like (class 1), blade-like (2), cuboid-like (3), plate-like (4) and cube-like (5). In general, the most accurate description of shape is achieved using the FC method given that features are unlikely to be orientated ideally within global coordinates. For the case of a cylindrical part built vertically along the main axis, the alignment of the boxing dimensions with global axes in the BB method allows a simple evaluation of whether a non-equidimensional feature is orientated towards the vertical (build) direction or horizontal scanning plane. This can be performed using an aspect ratio to distinguish features that are clearly elongated towards a particular direction. 


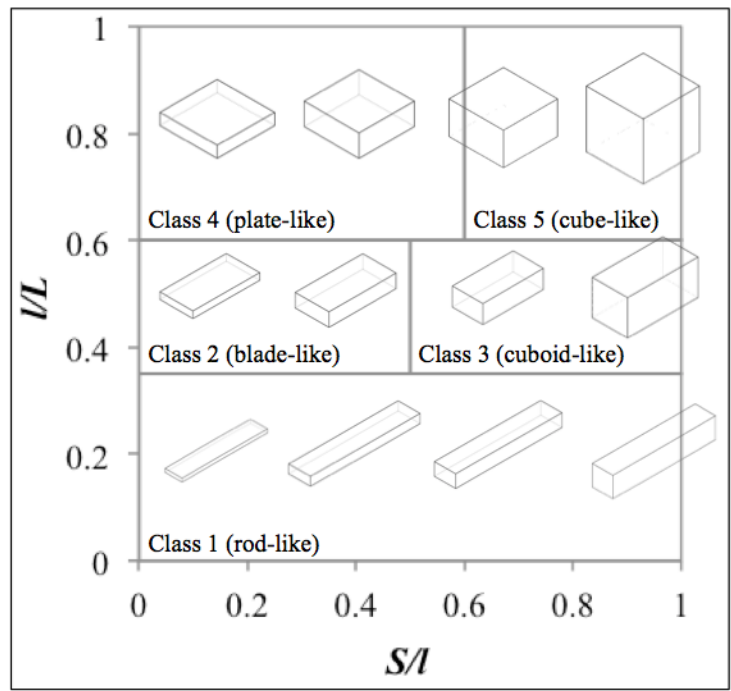

Figure 2. Pore shape classification based on dimensional descriptors. Adapted from $[27,28]$

\section{Results and Discussion}

\subsection{As-built Sample Results}

Results of the Avizo thresholding of as-built samples are given in Appendix B. Samples processed using identical parameters match reasonably closely. A maximum densification of $99.78 \%$ was achieved. The lowest was $68.64 \%$. Figure 3 shows porosity within the highest and lowest samples (3D rendered pores are displayed in random colours to distinguish individual features). Pores within samples of $<95 \%$ densification, produced using lowest power $(150 \mathrm{~W})$ and low $V E D\left(<45 \mathrm{~J} / \mathrm{mm}^{3}\right)$, were interconnected throughout the subvolume. Whilst in reality some separation of the porosity network may occur below the $2.96 \mu \mathrm{m}$ pixel size resolution, no attempt to manually separate pores was made to avoid misinterpretation of results (splitting a pore that exists as a single feature in reality). A comparison between porosities obtained using different $\mathrm{X} \mu \mathrm{CT}$ sub-volumes and $\mathrm{OM}$ for seven random samples is given in Table 3. The largest difference in porosities when comparing $\mathrm{X} \mu \mathrm{CT}$ sub-volumes is $0.56 \%$. The largest difference between $\mathrm{X} \mu \mathrm{CT}$ and OM was $2.20 \%$ for the most porous sample, with remaining differences being around $1 \%$ or less. This demonstrates reasonable consistency between methods when considering $\mathrm{OM}$ is particularly sensitive to slice location. Figure 4 shows a $2 \mathrm{D} \mathrm{X} \mu \mathrm{CT}$ radiograph and $\mathrm{OM}$ image for a sample.

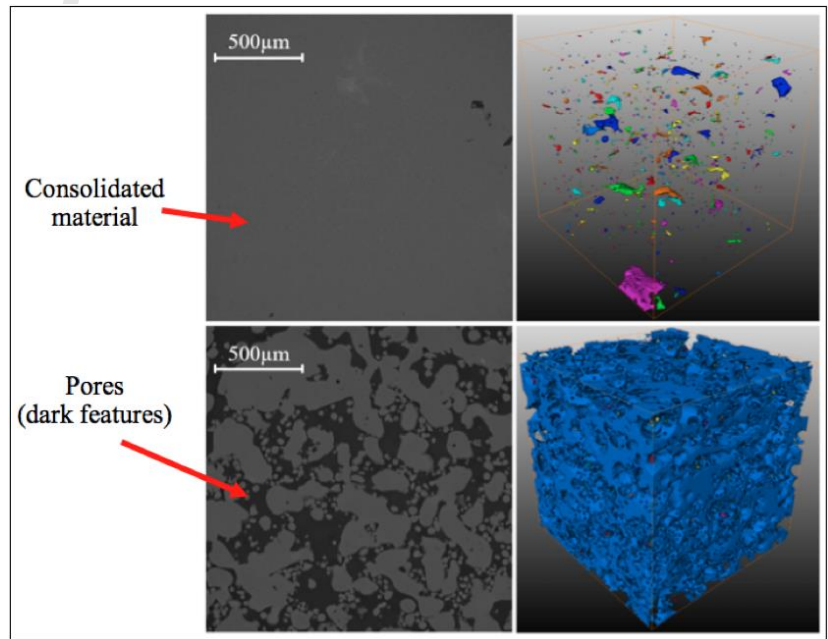

Figure 3. $\mathrm{X} \mu \mathrm{CT}$ radiographs and 3D sub-volume renderings of samples with highest (top) and lowest (bottom) as-built densifications 
Table 3. Comparison of $\mathrm{X} \mu \mathrm{CT}$ and $\mathrm{OM}$ as-built porosities

\begin{tabular}{|c|c|c|c|c|c|}
\hline \multirow{2}{*}{$\begin{array}{l}\text { Sample } P-v-h-t \\
(\mathrm{~W}-\mathrm{mm} / \mathrm{s}-\mu \mathrm{m}-\mu \mathrm{m})\end{array}$} & \multicolumn{2}{|c|}{ X $\mu \mathrm{CT}$ Porosity (\%) } & \multicolumn{2}{|c|}{ OM Porosity (\%) } & \multirow{2}{*}{$\begin{array}{l}\text { Max. absolute } \\
\text { difference between } \\
\text { methods }(\%)\end{array}$} \\
\hline & 600px cube & 500px cube & InfiniteFocus & Cerna & \\
\hline $250-500-75-60$ & 4.16 & 4.72 & 2.85 & 2.52 & 2.20 \\
\hline $250-2500-75-60$ & 3.45 & 3.80 & 2.76 & 2.92 & 1.04 \\
\hline $250-1500-45-60$ & 1.24 & 1.10 & 1.21 & 1.23 & 0.14 \\
\hline $150-500-45-60$ & 1.13 & 1.51 & 0.63 & 0.71 & 0.88 \\
\hline $250-1500-105-60$ & 1.10 & 1.41 & 2.00 & 2.11 & 1.01 \\
\hline $250-1500-105-30$ & 0.72 & 0.69 & 0.49 & 0.51 & 0.23 \\
\hline $350-500-45-60$ & 0.71 & 0.56 & 0.40 & 0.68 & 0.31 \\
\hline
\end{tabular}

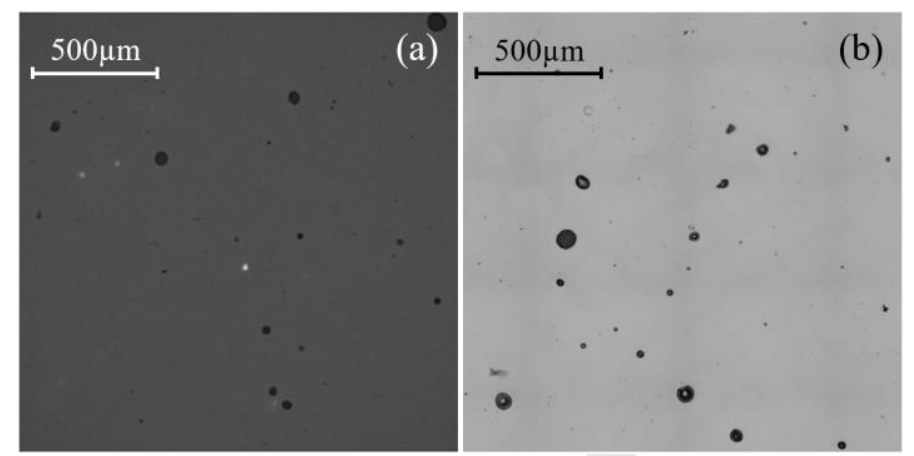

Figure 4. $\mathrm{X} \mu \mathrm{CT}$ (a) and InfiniteFocus $\mathrm{OM}$ (b) images (at different locations): ( $P=250 \mathrm{~W}, v=1500 \mathrm{~mm} / \mathrm{s}$, $h=105 \mu \mathrm{m}, t=30 \mu \mathrm{m})$

Laser energy density vs. as-built densification is shown in Figure 5 for all samples. Insufficient heat input leads to lack of layer fusion and failure to break down oxide films that exist in the powder and inhibit consolidation. At high scanning speeds, porosity is generated by melt flow instability and all combinations of lowest power $(150 \mathrm{~W})$ with highest speed $(2500 \mathrm{~mm} / \mathrm{s})$ produced densification $<95 \%$. All samples processed using 250 and $350 \mathrm{~W}$ powers exhibited densification greater than $95 \%$, as did $150 \mathrm{~W}$ samples above $48 \mathrm{~J} / \mathrm{mm}^{3}$. The optimum densification $\left(99.78 \%\right.$ ) was achieved using $222 \mathrm{~J} / \mathrm{mm}^{3}$ with $P=150 \mathrm{~W}, v=500 \mathrm{~mm} / \mathrm{s}, h=45 \mu \mathrm{m}$ and $t=30 \mu \mathrm{m}$, i.e. the lowest power, slowest speed and smallest spacing and thickness. The same combination but with $350 \mathrm{~W}$ power produced a lower densification of $98.96 \%$, attributed to overheating causing evaporation and oxidation. We note the miniscule difference between the highest and second highest results ( 99.78 and $99.77 \%$ ) is well within the realms of post-processing error, with the latter achieved using only $95 \mathrm{~J} / \mathrm{mm}^{3}$. Table 4 compares some energy densities and corresponding densifications reported for $\mathrm{AlSi} 10 \mathrm{Mg} / \mathrm{Al}-\mathrm{Si}$ alloys from the literature, where different processing parameters and scanning strategies were employed. A $V E D$ of $222 \mathrm{~J} / \mathrm{mm}^{3}$ is substantially greater than reported values for achieving high densification, while $95 \mathrm{~J} / \mathrm{mm}^{3}$ is more in line with previous studies. In practical terms, there is no direct correlation that can be used to optimise the LPBF build in terms of densification as porosity is sensitive to melt pool behaviour governed by processing parameters and powder absorptivity will vary depending on heat input [31]. Moreover, in Table 4 we see how Tradowsky et al. [16] and Larrosa et al. [17] obtained relatively low densification when processing according to parameters optimised in [5] for different powder morphology. Two other samples processed here using $222 \mathrm{~J} / \mathrm{mm}^{3}$ but different parameter combinations exhibited low densifications of 96.61 and $96.74 \%$ (see Appendix B), further highlighting the importance of treating energy density as a 'rule-of-thumb'. 
Table 4. Comparison of laser energy densities and densifications in AlSi10Mg/Al-Si alloys processed using various parameters and scanning strategies

\begin{tabular}{rrr}
\hline Study & VED (J/mm $\left.\mathbf{m}^{\mathbf{3}}\right)$ & Densification (\%) \\
\hline Present work & $222(95)$ & $99.78(99.77)$ \\
Wang et al. [30] & 131 & $99.75^{+}$ \\
Aboulkhair et al. [3] & $100^{*}$ & 99.82 \\
Cai et al. [13] & 93.33 & $99.31^{+}$ \\
Olakanmi [21] & $60-75$ & - \\
Read et al. [5] & 60 & - \\
Tradowsky et al. [16] & $58.37^{*}$ & $98.3^{+}$ \\
Larrosa et al. [17] & $58.37^{*}$ & $98.0^{+}$ \\
Thijs et al. [2] & $45^{*}$ & 99.4 \\
\hline * - VED calculated using Equation (3) based on reported parameters \\
${ }^{+}$- Densification inverted from reported minimum porosity
\end{tabular}
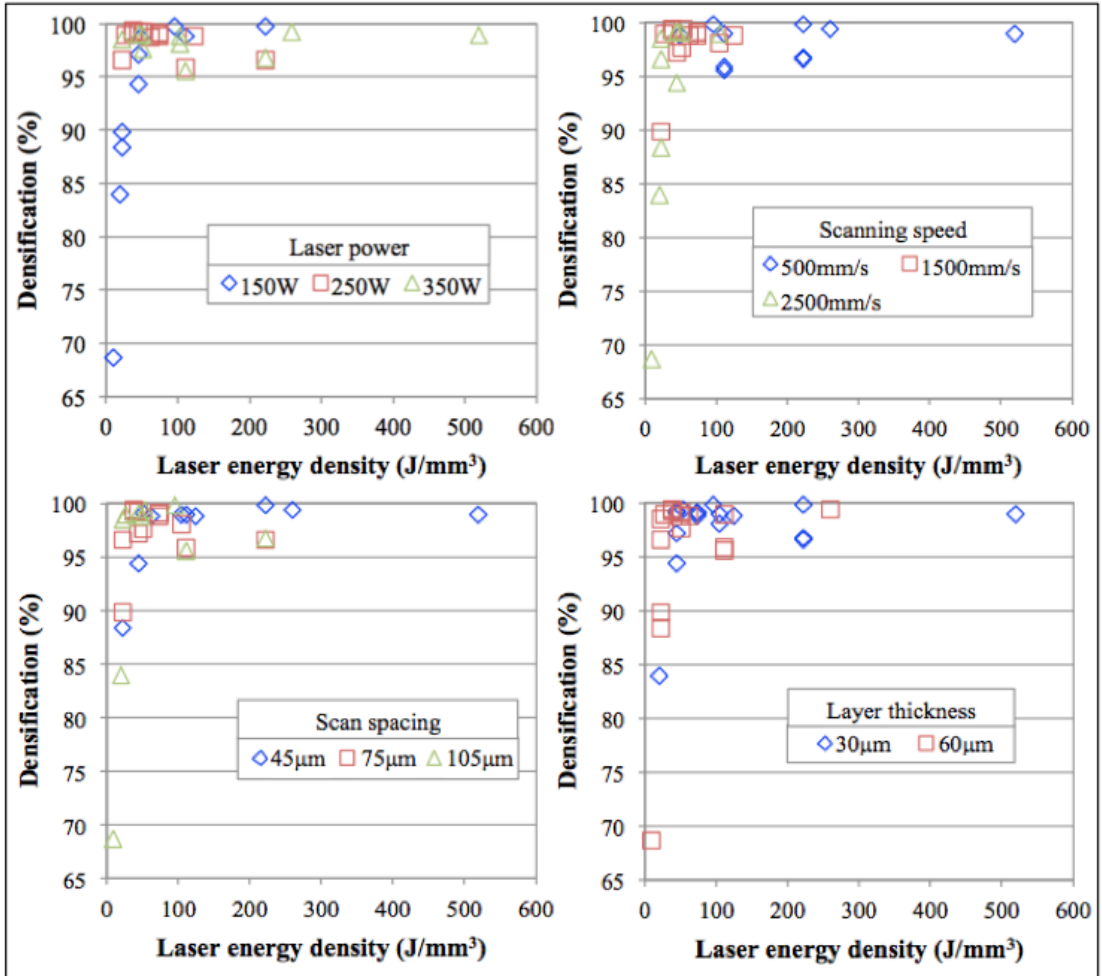

Figure 5. Laser energy density vs. as-built densification showing key processing parameters (figure available in colour online)

\subsection{As-built Pore Shape Analysis}

To better understand the influence of laser energy density on porosity we consider pore shape. Pore size is categorised according to Table 5. The relationship between energy density and average sphericity for small, medium and large pores, excluding samples containing interconnected porosity, is shown in Figure 6. Very small pores are not considered as an accurate sphericity for features approaching $1 \mathrm{px}$ cannot be determined using Avizo $(\Psi>1.0)$. However, we observe that decreasing size is generally accompanied by increasing average sphericity. In samples processed using 250 and $350 \mathrm{~W}$ laser powers the average sphericity increases to near 1.0 above a threshold occurring between $26-37 \mathrm{~J} / \mathrm{mm}^{3}$. This increase is significant for medium and large sizes $\left(d_{e q}>100 \mu \mathrm{m}\right)$ as the dominant defect type is seen to switch from lack of fusion defects to gas porosity above the threshold. Above $222 \mathrm{~J} / \mathrm{mm}^{3}$ there appears to be a slight decrease in sphericity that 
suggests melt pool overheating and keyhole instability by excessive energy input. By comparison, pores within samples processed by $150 \mathrm{~W}$ exhibit significantly lower average sphericity and sphericity does not vary appreciably with energy density. In other words, low power results in lack of fusion and this does not change when raising the energy density by lowering scanning speed and/or reducing spacing/thickness. Figure 7 shows examples of a spherical gas pore and irregular fusion defect. The samples were processed using comparable $V E D$ but high and low powers.

Table 5. Pore size categorisation

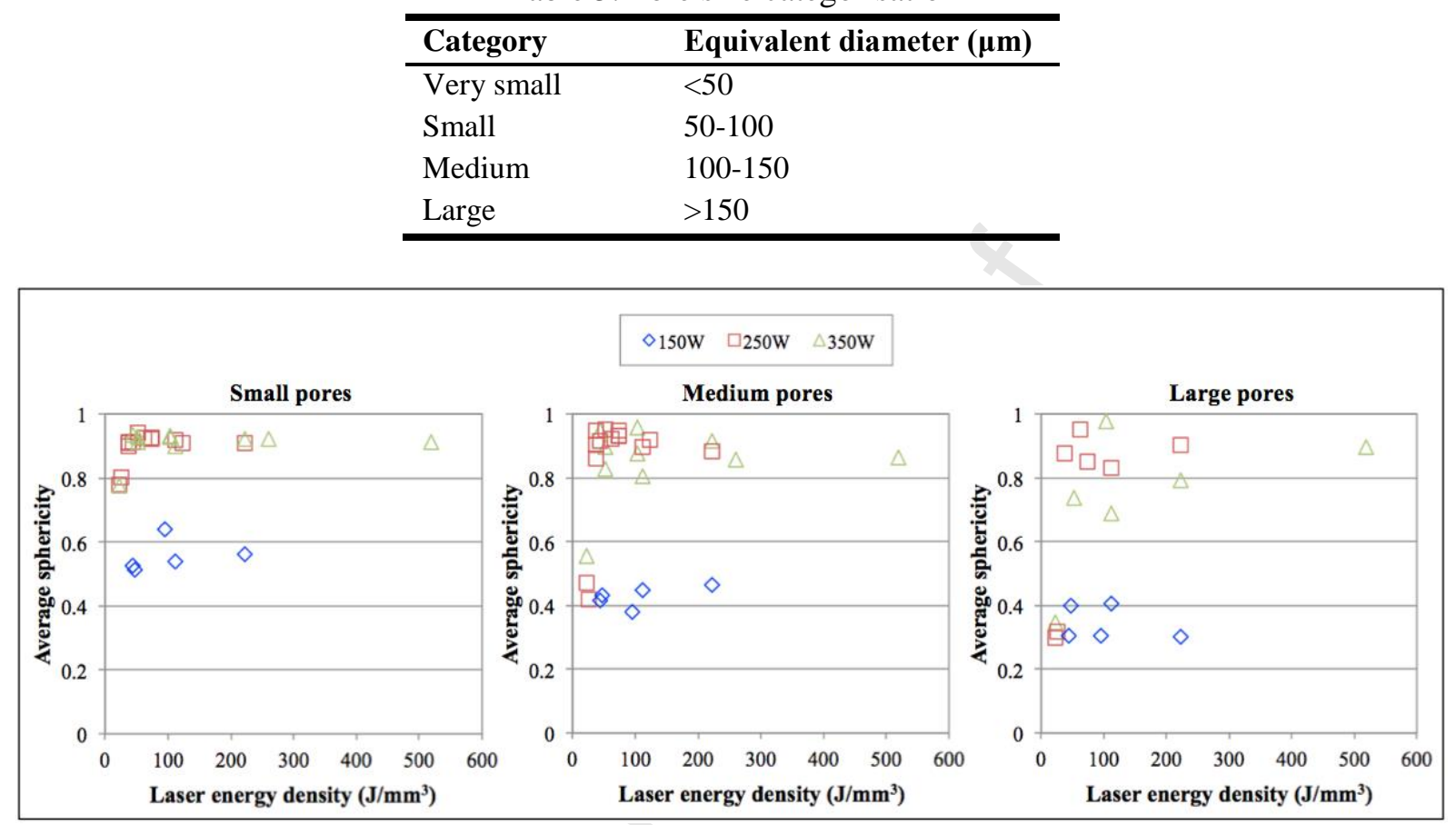

Figure 6. Laser energy density vs. average sphericity for small, medium and large pores (figure available in colour online)

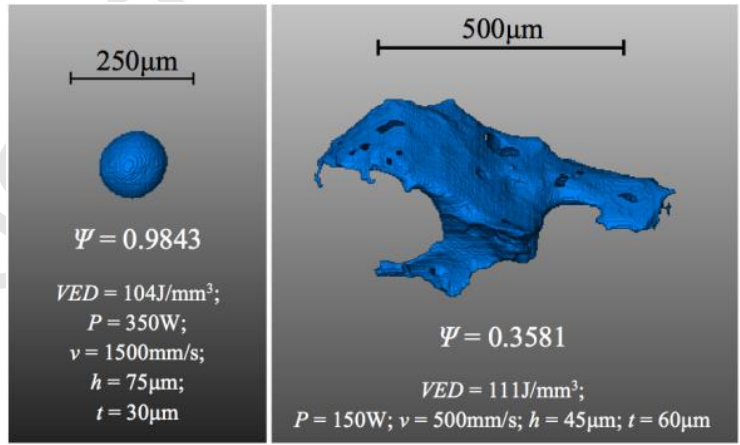

Figure 7. Examples of highly spherical gas pore (left) and irregular fusion defect (right)

Example pore classification diagrams based on FC and BB descriptors for samples with high and low average pore sphericities are shown in Figure 8. In addition to the basic distribution, a second BB chart is plotted showing pores that are bound with aspect ratio $\geq 1.5$ in either the horizontal scanning plane $(X Y)$ or vertical build direction $(Z)$. A factor of 1.5 is chosen to avoid labelling near equidimensional pores as directionally biased. In Figure 8a, the FC and BB methods agree relatively well for small and medium pores in and around class 5 . With predominantly spherical pores there is little directional bias; $5 \%$ of small pores are horizontally orientated by factor $\geq 1.5$ and $<1 \%$ (a single pore) is vertically orientated. There is greater discrepancy between methods for the sample containing highly irregular pores shown in Figure $8 \mathrm{~b}$. Using the 
FC method, which accounts for orientation in determining 3D descriptors, pores are clustered at the intersections between classes 2-5 (blade, cuboid, plate and cube respectively). Conversely, pores are more widely spread across the same classes in the BB distribution. We observe from the BB analysis that large proportions of all pore sizes are significantly elongated in the horizontal plane, orthogonal to the build direction and parallel to the laser scanning. Formation of irregular lack of fusion defects with long axis lying perpendicular to the building direction is typically expected with low energy processing [16-19]. Here, we see these defects occupy blade-, cuboid-, plate- and (flattened) cube-like 3D spaces based on the FC descriptors.

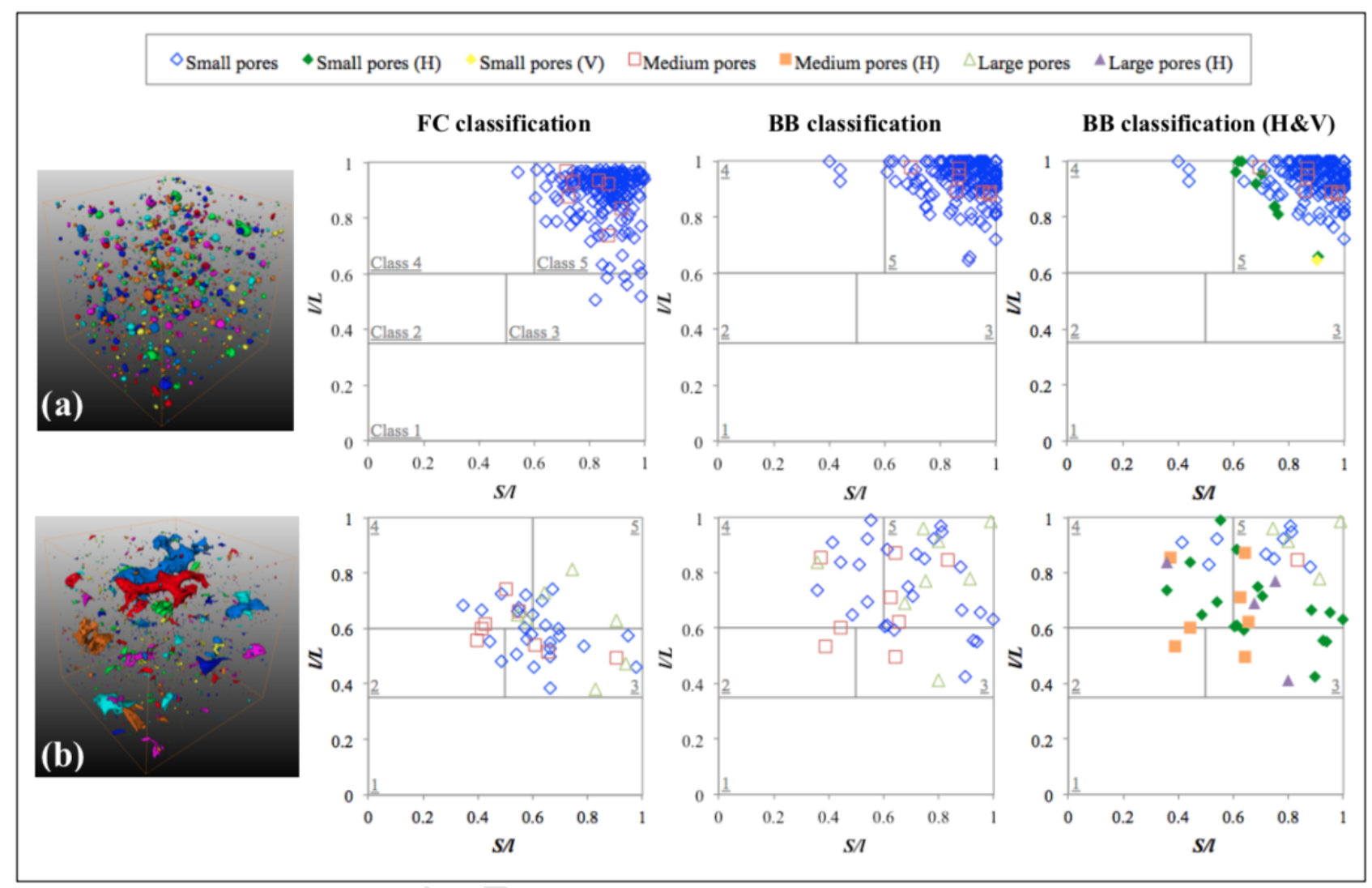

Figure 8. Pore FC and BB classifications for as-built samples with high and low average sphericities (figure available in colour online): $P=250 \mathrm{~W}, v=1500 \mathrm{~mm} / \mathrm{s}, h=75 \mu \mathrm{m}, t=30 \mu \mathrm{m}(\mathrm{a}) ; P=150 \mathrm{~W}, v=500 \mathrm{~mm} / \mathrm{s}, h=105 \mu \mathrm{m}$, $t=60 \mu \mathrm{m}(\mathrm{b})$. Directionality in the BB method classified by horizontal $(\mathrm{H})$ or vertical $(\mathrm{V})$ aspect ratio $\geq 1.5$

The FC class frequencies of small, medium and large pores within sample sub-volumes in as-built condition, excluding samples with interconnected porosity, are given in Figures 9-11. The majority of small pores are class 3 (cuboid-like) followed by class 4 (plate-like) in all samples processed using 150W. At higher scanning speed there is a rise in pore quantity and we recall that samples containing interconnected porosity, omitted from Figures 9-11, were processed using 150W namely in conjunction with high speeds. There is an increase in the proportion of class 5 with increasing pore size, although as seen earlier the sphericity becomes especially low. Therefore, all energy densities comprising $150 \mathrm{~W}$ power used here produced samples containing either extreme interconnected porosity or less-severe porosity volumes comprised primarily of isolated cuboid- and plate-like small pores with larger irregular pores being proportionally more equidimensional, i.e. occupying a relatively cubic 3D space. On the other hand, small pores in all samples processed using 250 and $350 \mathrm{~W}$ powers are predominantly class 5 and mainly spherical gas pores, as shown previously. For medium and large sizes there is an increase in the proportion of nonequidimensional pores. Again, there is a higher count of class 3 than class 4, meaning non-equidimensional 
pores are more commonly elongated in one of three dimensions than two of three overall for the parameter range studied here.

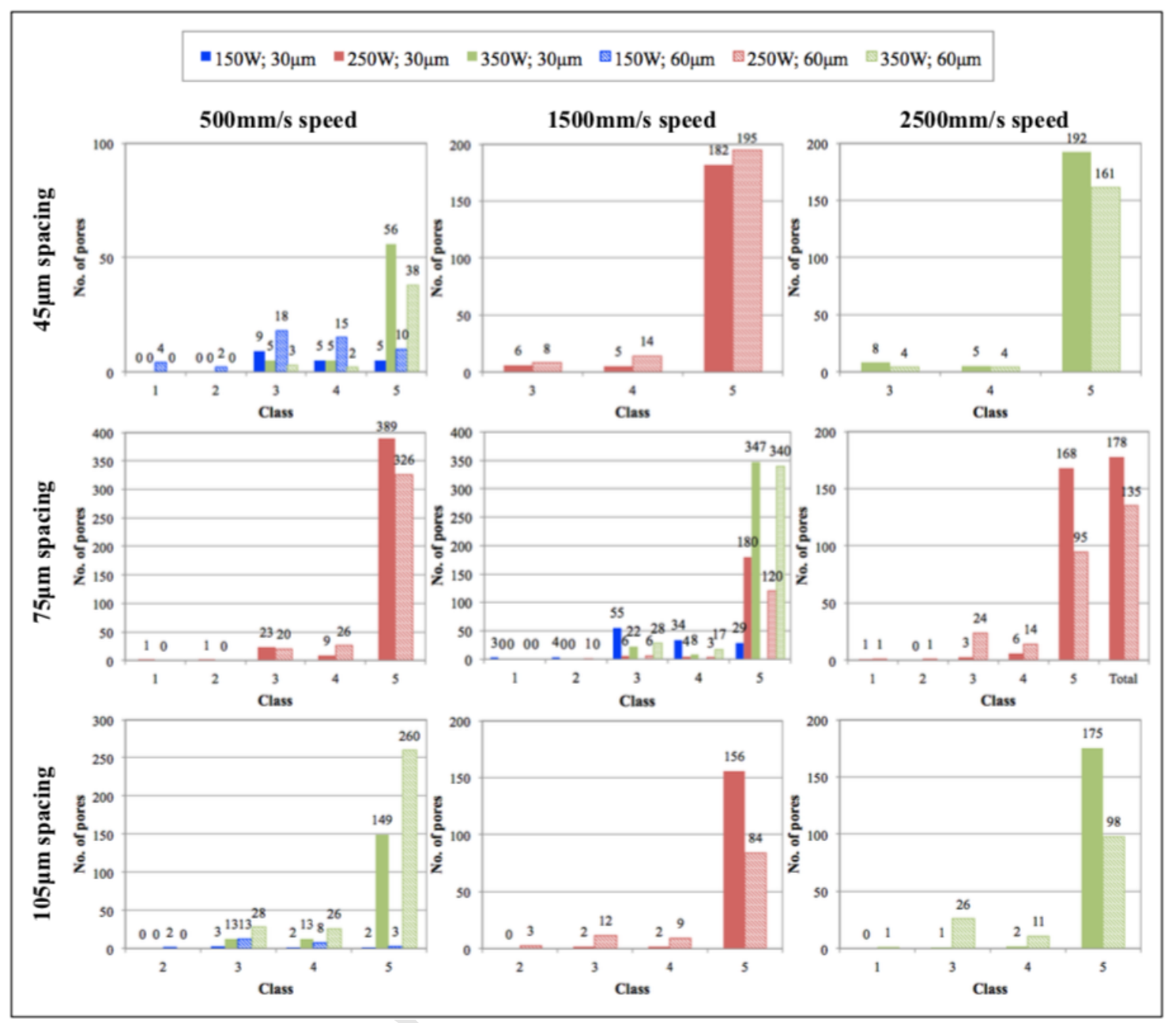

Figure 9. Small pore FC class frequencies in as-built samples. Sample designation: $P ; t$ (figure available in colour online) 


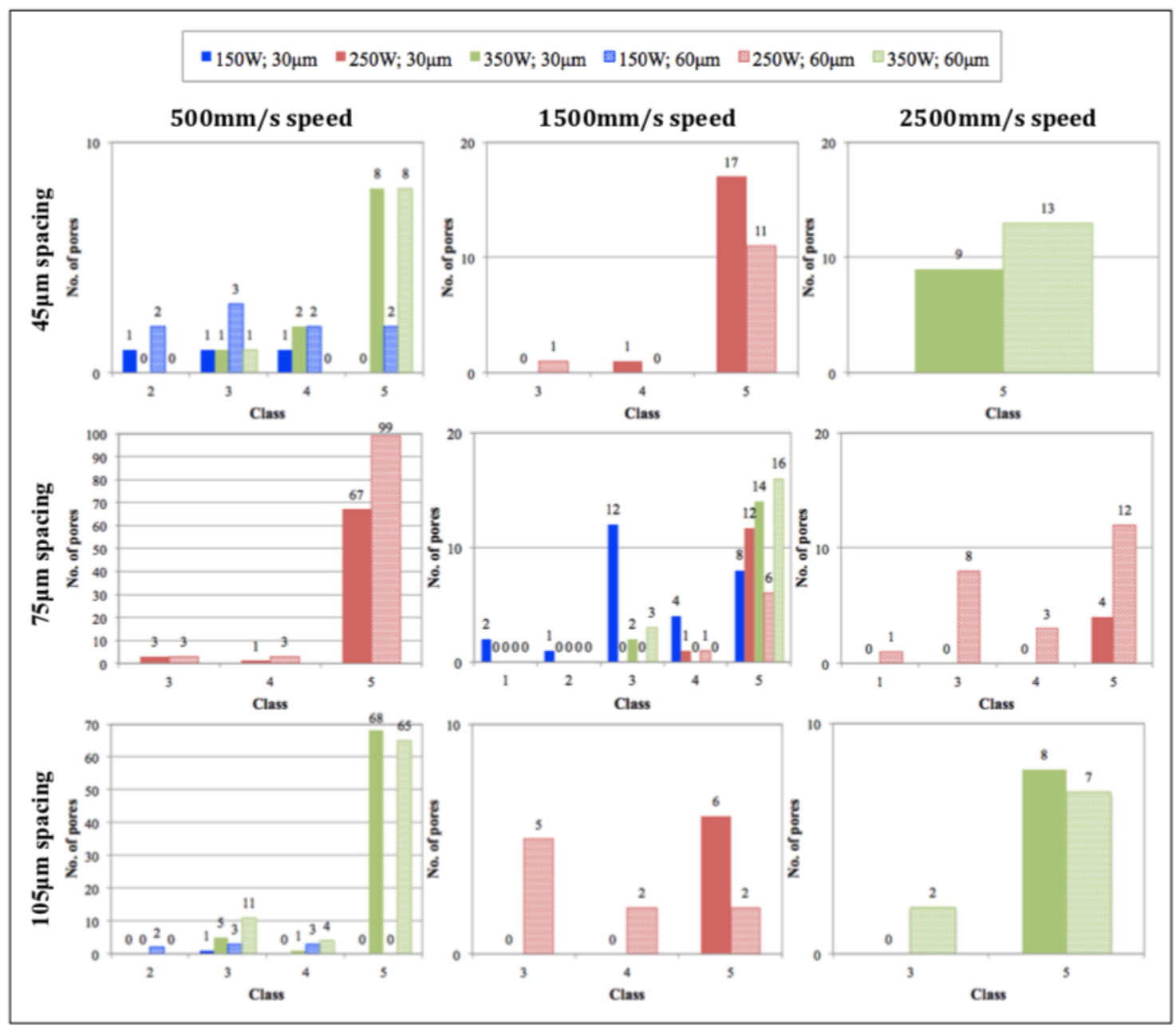

Figure 10. Medium pore FC class frequencies in as-built samples. Sample designation: $P ; t$ (figure available in colour online)

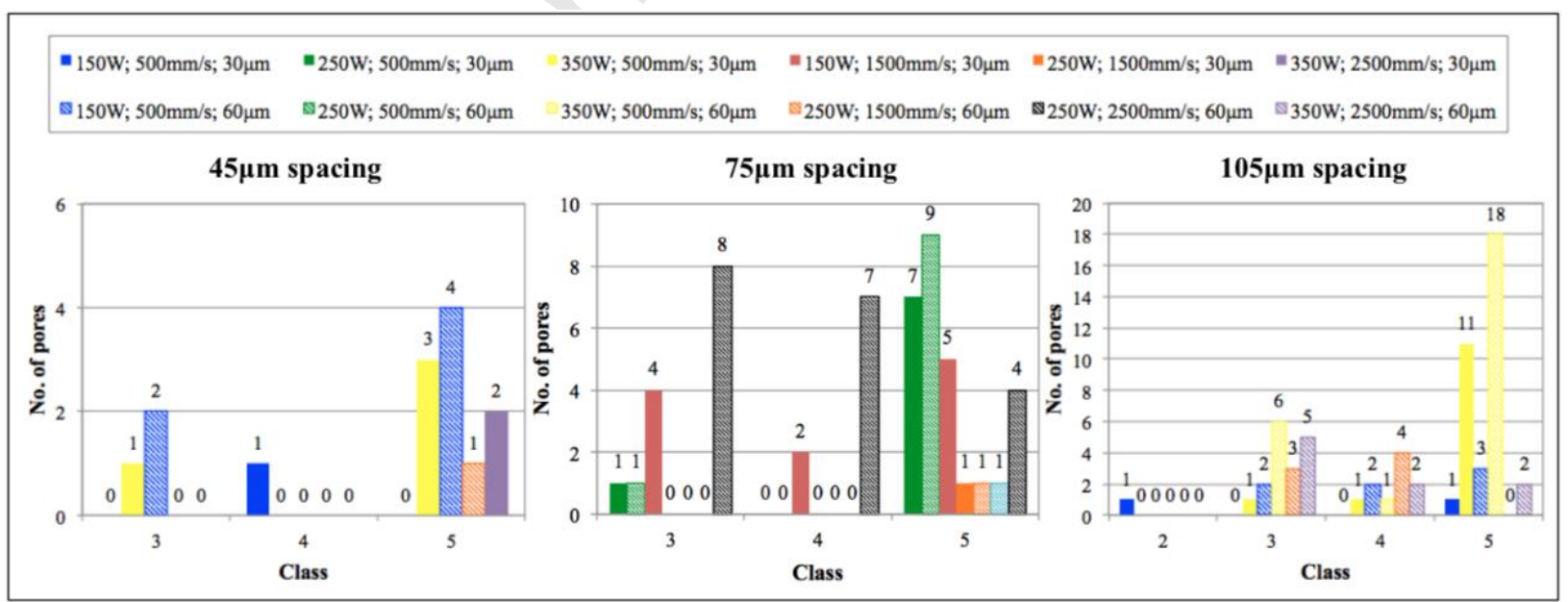

Figure 11. Large pore FC class frequencies in as-built samples. Sample designation: $P ; v ; t$ (figure available in colour online)

Figures 12-14 show the relative frequencies of pores with horizontal and vertical BB orientations within non-interconnected porosity samples containing at least five individual pores of each size. An aspect ratio $\geq 1.5$ is again chosen to distinguish clearly elongated pores. Significantly more pores are elongated horizontally in the scanning plane than vertically in the build direction. All parameter combinations with 
150W power yielded a high proportion of horizontal pores; the lowest relative frequencies for small, medium and large are 57.1, 67.9 and 50.0\% respectively. From the FC analysis, we know that the majority of these are long and narrow as opposed to long and wide. It must be reiterated that the BB approach is inherently binary in that the only distinguished directions are vertical (in the build direction) or transverse and therefore pore axes do not necessarily align perfectly with the vertical axis or horizontal plane. In general, relative frequency does not change drastically with pore size for $150 \mathrm{~W}$ and there is no clear correlation between energy density and the frequency of highly directional pores.

For $250 \mathrm{~W}$, the highest horizontal relative frequencies for all pore sizes are observed in two samples processed using very low $V E D\left(\leq 26 \mathrm{~J} / \mathrm{mm}^{3}\right)$ and proportionality increases with size. For example, processing with $22 \mathrm{~J} / \mathrm{mm}^{3}(P=250 \mathrm{~W}, \nu=2500 \mathrm{~mm} / \mathrm{s}, h=75 \mu \mathrm{m}, t=60 \mu \mathrm{m})$ resulted in $25.9,50.0$ and $90.5 \%$ of small, medium and large pores being orientated in the scanning plane respectively. For $350 \mathrm{~W}$, the highest proportions of all sizes were observed in the sample processed using similarly low $V E D\left(22 \mathrm{~J} / \mathrm{mm}^{3}\right)$ although the increase in relative frequency with size is less drastic and pores are less frequently elongated. Low relative frequencies were produced by all 250 and $350 \mathrm{~W}$ parameter combinations yielding $V E D \geq 37 \mathrm{~J} / \mathrm{mm}^{3}$ (maximum 10.2, 30.0 and $30.0 \%$ for small, medium and large respectively). At $37 \mathrm{~J} / \mathrm{mm}^{3}$ and above there is no clear trend between energy and pore directionality for 250 and $350 \mathrm{~W}$ samples. Small, medium and large vertically orientated pores are most evident in samples processed using 250 and 350W and exceptionally large $V E D\left(519 \mathrm{~J} / \mathrm{mm}^{3}\right)$, indicating keyhole instability, or high speed, particularly in conjunction with large spacing and thickness (melt pool turbulence with minimal overlap).

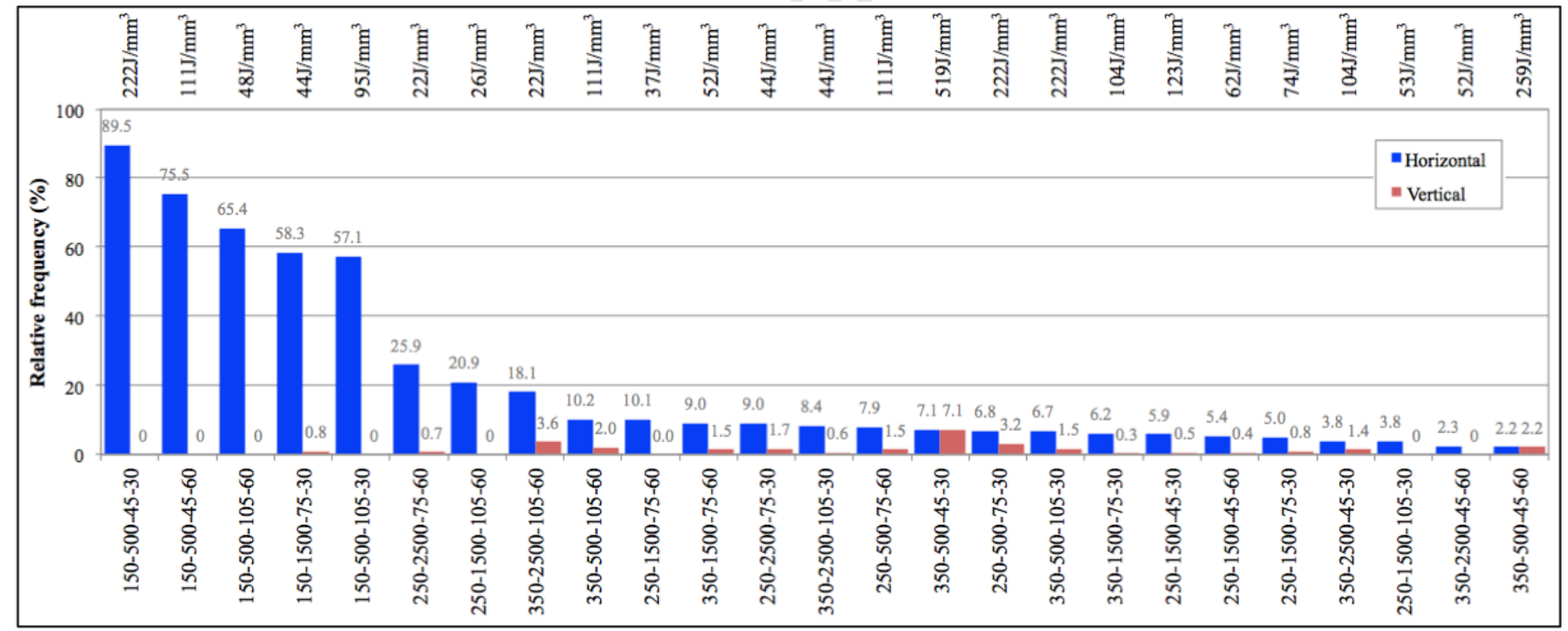

Figure 12. Relative frequency of horizontal and vertical small pores with $\mathrm{BB}$ aspect ratio $\geq 1.5$ (figure available in colour online). Sample designation: $P-v-h-t(\mathrm{~W}-\mathrm{mm} / \mathrm{s}-\mu \mathrm{m}-\mu \mathrm{m})$ 


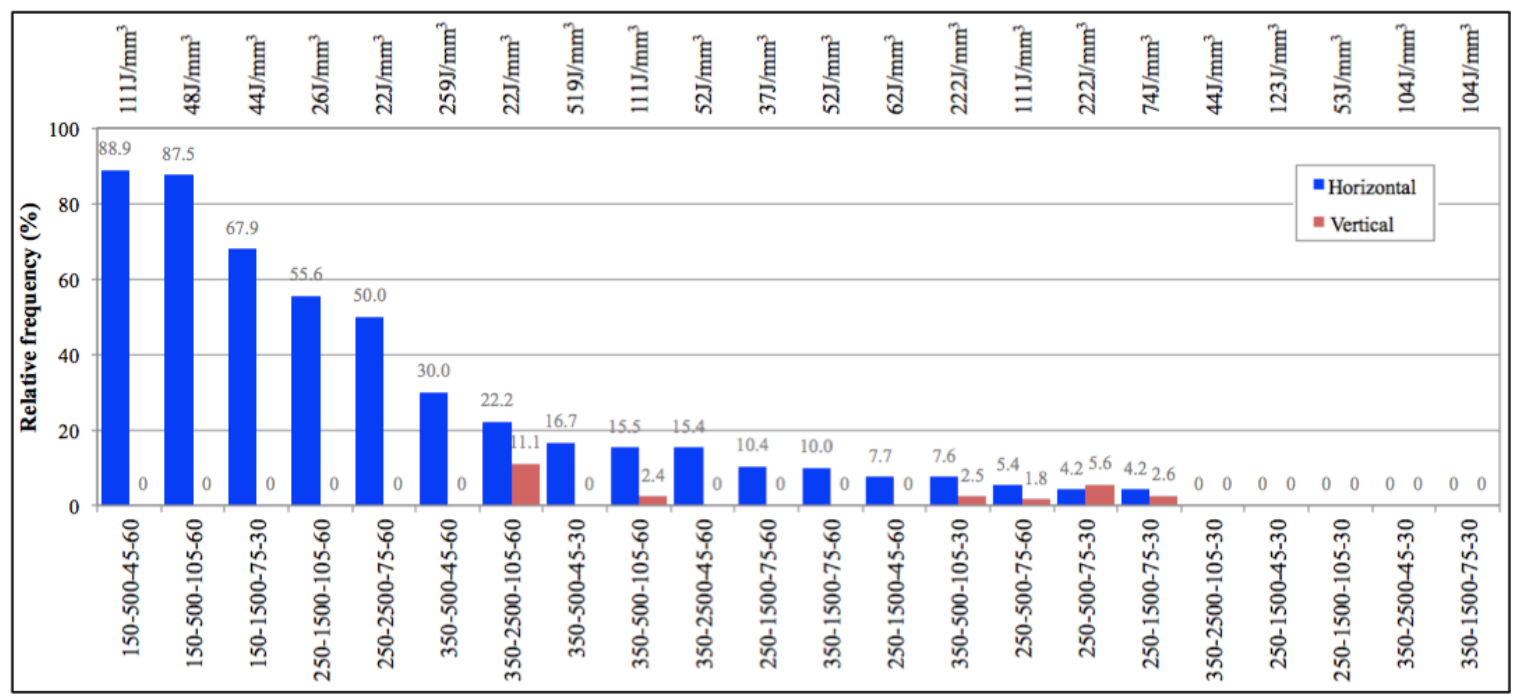

Figure 13. Relative frequency of horizontal and vertical medium pores with $\mathrm{BB}$ aspect ratio $\geq 1.5$ (figure available in colour online). Sample designation: $P-v-h-t(\mathrm{~W}-\mathrm{mm} / \mathrm{s}-\mu \mathrm{m}-\mu \mathrm{m})$

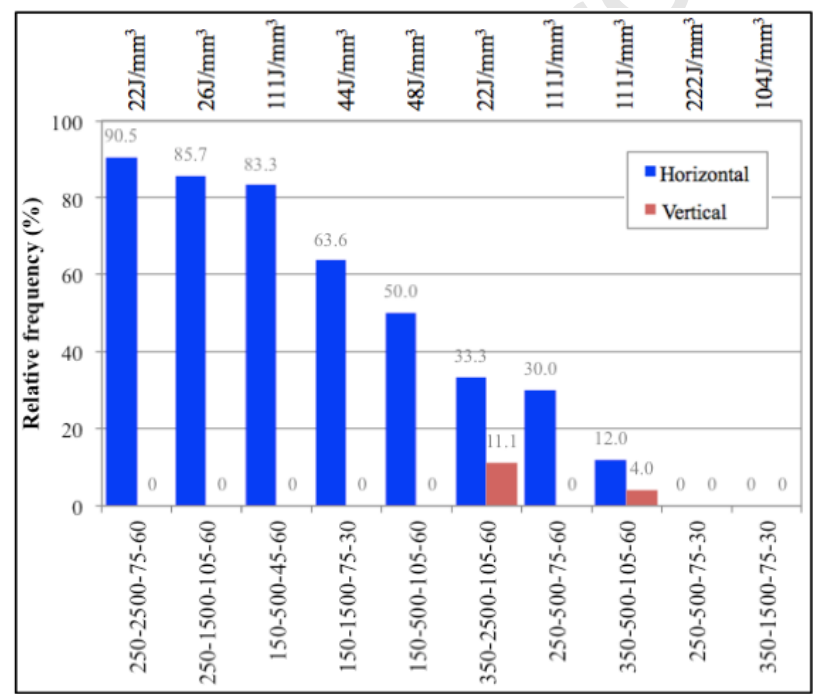

Figure 14. Relative frequency of horizontal and vertical large pores with $\mathrm{BB}$ aspect ratio $\geq 1.5$ (figure available in colour online). Sample designation: $P-v-h-t(\mathrm{~W}-\mathrm{mm} / \mathrm{s}-\mu \mathrm{m}-\mu \mathrm{m})$

\subsection{Effects of Heat Treatment}

Based on the as-built study the two highest and two lowest densification samples were subjected to HIPping followed by $\mathrm{T} 6$, along with a further two samples processed using identical parameters to assess repeatability. A summary of Avizo thresholding results for treated samples is given in Appendix B. Densifications of samples in different states are shown in Figure 15. The morphology of interconnected porosity within low as-built densification samples did not change appreciably with treatment, as can be seen for the most porous sample in Figure 16. However, HIPping collapsed pores isolated within consolidated regions manifesting in a sizeable reduction in the quantity of detected pores greater than $1 \mathrm{px}$. Pores were reopened by T6 although not to the as-built extent. Pore closure and reopening appears inconsistent with a drop in HIPped densification measured for the lowest densification sample. It is possible the drop is associated with thresholding error or imprecision when rescanning the sample and matching features to relocate the sub-volume, which is especially difficult for highly porous bodies. In any case, treatment was ineffective at closing interconnected porosity within very low densification samples (Figure 16). Porosity in samples exhibiting high as-built densification was reduced to virtually negligible by HIPping, which was 
also observed by Tradowsky et al. [16] and Larrosa et al. [17] for less optimal builds ( $<98.5 \%$ densification). Porosity then increases with T6 but remains below the as-built level.

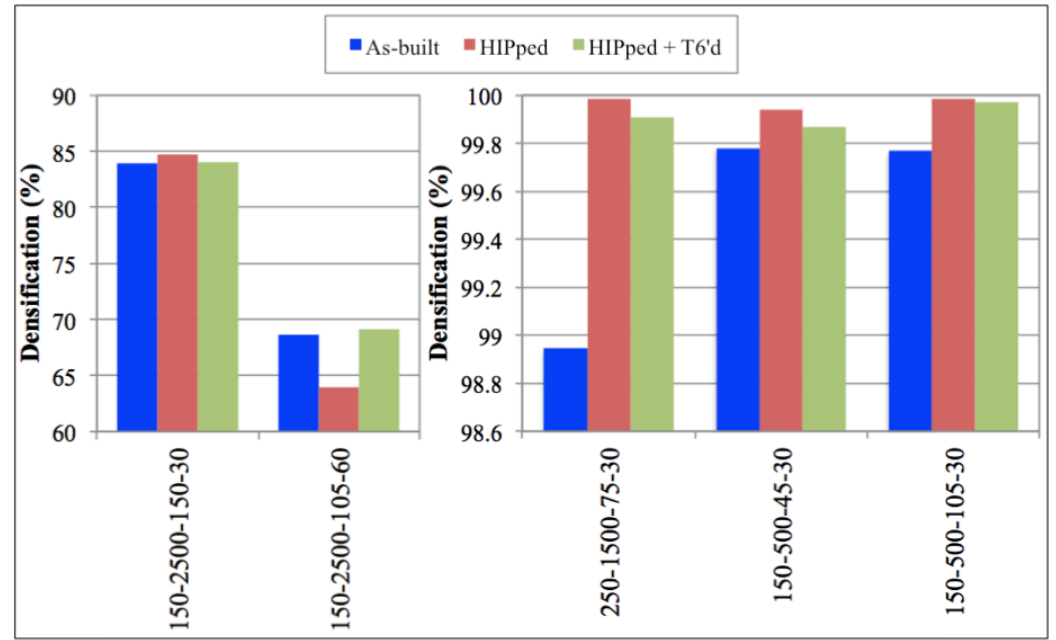

Figure 15. Densification of low (left) and high (right) as-built samples in different states (figure available in colour online)
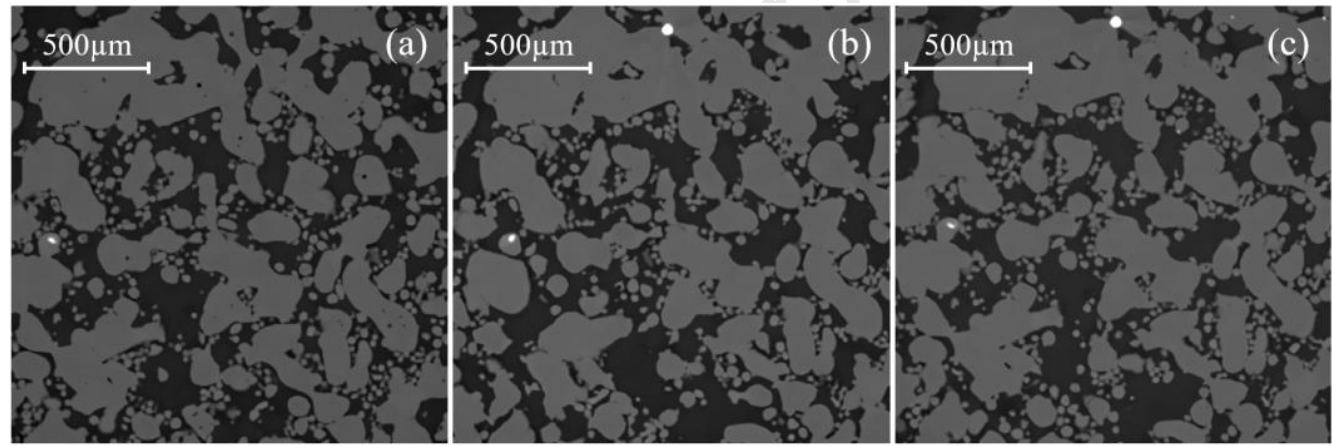

Figure 16. X $\mu \mathrm{CT}$ radiographs $(P=150 \mathrm{~W}, \nu=2500 \mathrm{~mm} / \mathrm{s}, h=105 \mu \mathrm{m}, t=60 \mu \mathrm{m})$ showing no change in interconnected porosity (dark regions) with treatment: as-built (a); HIPped (b); HIPped + T6'd (c)

Classification diagrams based on the FC method are shown in Figure 17 for high densification samples in different states. Very small pores that exhibit $\Psi<1.0$ are included simply to demonstrate that porosity remains following treatment, although we note that measurements approaching 1px on Avizo are inherently unreliable. In Figure 17a and b showing samples processed using identical parameters, pores with $d_{e q}>50 \mu \mathrm{m}$ are collapsed by HIPping and remain closed post-T6 with the exception of a single small pore that remains after both stages (Figure 17b). The majority of collapsed as-built pores are in or around class 5 . The remaining pore is isolated in class 4 post-HIPping but returns to class 5 following T6 (shown as a solid yellow triangle for clarity). This suggests a partial HIPping collapse causing a flattening of shape (plate-like) and subsequent re-expansion by T6. It is not clear why there is incomplete closure. Kan et al. [19] found that HIPping could not collapse pores within AlSi10Mg builds exhibiting 96\% densification, which is between the optimal and extreme low levels studied here. They suggested porosity remained open due to entrapped argon. This may be the cause of incomplete closure in this case given that the pore exhibits high sphericity and is indicative of a gas defect, which might be difficult to close depending on the gas solubility [32]. Partial collapse may also be influenced by build direction, as the effectiveness of HIPping on specimens was found to differ depending on vertical or horizontal building by Larrosa et al. [17]. Interestingly, in the two identically processed samples we observe a slight increase in very small class 3 pores with HIPping and in Figure $17 \mathrm{~b}$ there is the notable appearance of class 1 pores, which also implies partial collapse and shape 
alteration. However, this can only be speculated given the aforementioned uncertainty with very small features. Despite inability to accurately quantify shape, the presence of very small pores post-treatment is clear and one can deduce that these are highly spherical in the as-built condition (which agrees with the distribution) considering a general increase in average sphericity with decreasing pore size (evident in Figure 6) and thus associated with entrapped gas. HIPping may therefore be somewhat ineffective at completely collapsing very small gas pores.

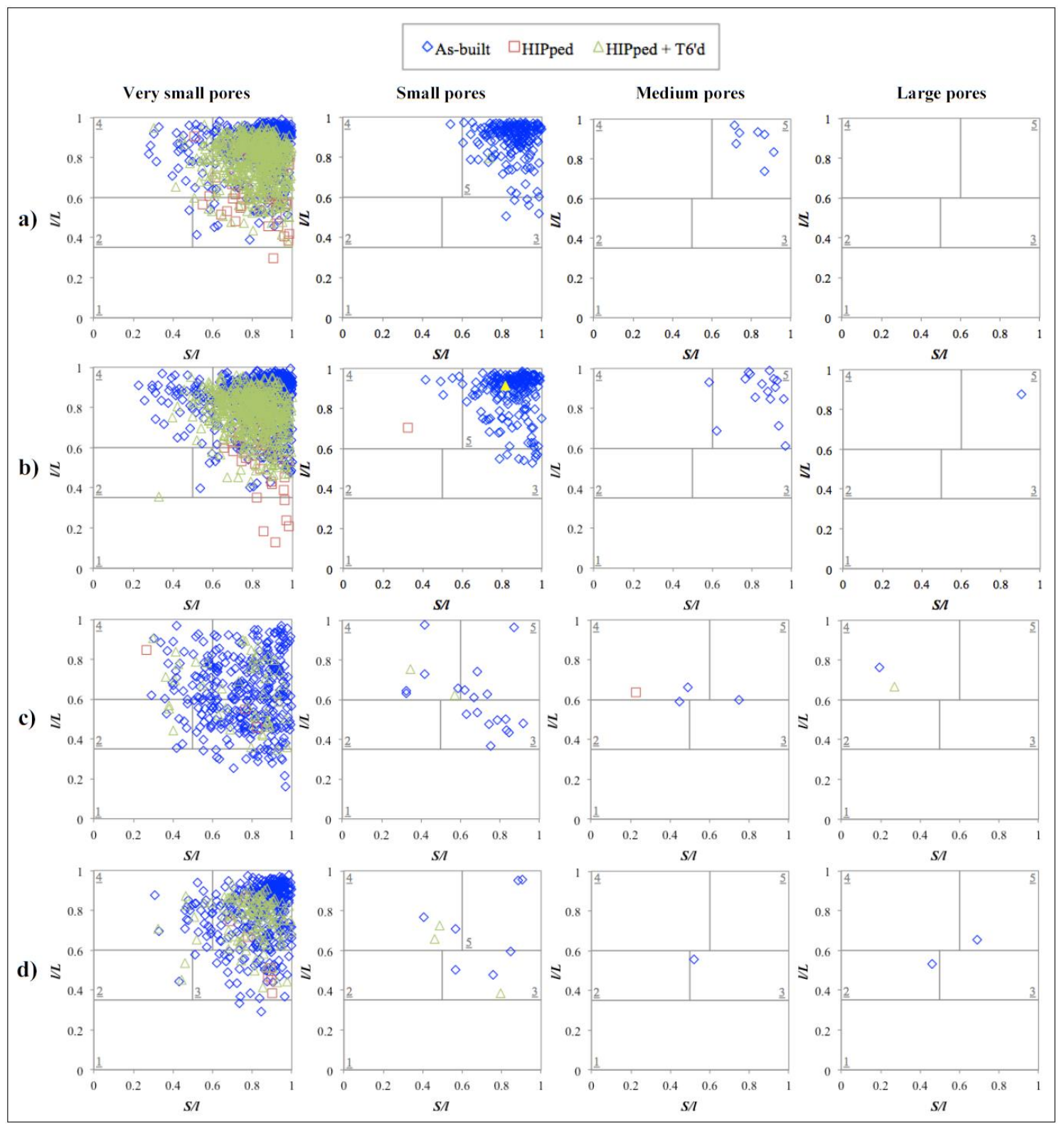

Figure 17. FC classification of pores in as-built and treated states (figure available in colour online): $P=250 \mathrm{~W}, v=1500 \mathrm{~mm} / \mathrm{s}, h=75 \mu \mathrm{m}, t=30 \mu \mathrm{m}(\mathrm{a} / \mathrm{b}) ; P=150 \mathrm{~W}, v=500 \mathrm{~mm} / \mathrm{s}, h=45 \mu \mathrm{m}, t=30 \mu \mathrm{m}(\mathrm{c}) ; P=150 \mathrm{~W}$, $\nu=500 \mathrm{~mm} / \mathrm{s}, h=105 \mu \mathrm{m}, t=30 \mu \mathrm{m}(\mathrm{d})$

In Figure 17c, there appears to be incomplete collapse of a large pore that drops into the medium size category after HIPping but is reopened by T6, yet the shape classification does not change drastically with the drop in size. This pore was located at the sub-volume boundary and only partly visible within the analysis, as shown in Figure 18. The equivalent diameter was much larger than all other pores isolated within treated samples $\left(d_{e q}=346.45 \mu \mathrm{m} ; 171.91 \mu \mathrm{m}\right.$ inside the sub-volume). In actuality, morphology was not appreciably altered and the measured change in size is likely to be imprecision in realigning sub-volume 
boundaries after rescanning. Closure of other pores of regular and irregular shape (with the exception of the aforementioned small pore) demonstrates the effects of HIPping depend on pore size. This follows nonclosure of interconnected porosity shown previously where gas content or lack of sufficient surrounding material may render porosity uncollapsible by diffusion and small scale plastic flow mechanisms. In Figure $17 \mathrm{c}$ and d, HIPping effectively closed all other pores with $d_{e q}>50 \mu \mathrm{m}$ scattered across classes 3-5. This indicates the effects are not sensitive to shape and formation mechanism, which may include oxidation as shown by Tradowsky et al. [16], although any oxide layers are expected to remain in the treated material. The shapes of class 3 and 4 pores reopened by T6 resemble those in the as-built state albeit the data points do not match perfectly, i.e. pores reopen to roughly the as-built shape.

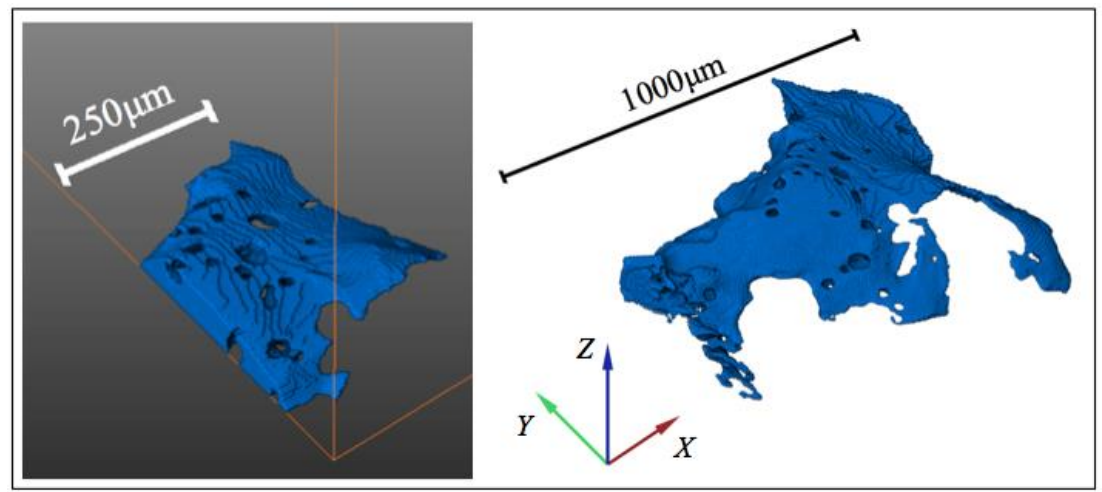

Figure 18. Very large pore (shown in as-built condition) located at cubic sub-volume boundary that was not altered by treatment $(P=150 \mathrm{~W}, v=500 \mathrm{~mm} / \mathrm{s}, h=45 \mu \mathrm{m}, t=30 \mu \mathrm{m})$

\section{Conclusions}

In the present paper, AlSi10Mg samples were manufactured by LPBF using 30 combinations of laser power, scanning speed, scan spacing and layer thickness, covering a range of densifications. Samples were scanned by high resolution $\mathrm{X} \mu \mathrm{CT}$ to quantify porosity using an approach based on simplified 3D object classes. A $V E D$ of $222 \mathrm{~J} / \mathrm{mm}^{3}$ produced the optimal densification $(99.78 \%)$ but also relatively low densifications (96.74 and $96.61 \%$ ). A near identical optimum $\left(99.77 \%\right.$ ) was produced using only $95 \mathrm{~J} / \mathrm{mm}^{3}$, demonstrating how $V E D$ alone cannot be used to optimise densification.

Processing with $150 \mathrm{~W}$ at high speeds produced interconnected porosity. Samples processed using $150 \mathrm{~W}$ at higher $V E D$ contained separated pores predominantly formed by lack of fusion and elongated in the scanning plane irrespective of $V E D$ and size. These primarily occupied a cuboid space. For 250 and 350W, average sphericity increased above a threshold occurring between $26-37 \mathrm{~J} / \mathrm{mm}^{3}$, indicating a switch in dominant formation mechanism from poor fusion to gas trapping, and decreased above $222 \mathrm{~J} / \mathrm{mm}^{3}$ due to overheating. The proportion of horizontally biased pores was greater in 250 and $350 \mathrm{~W}$ samples processed at low $V E D$ than at high $V E D$ and relative frequency varied with size. Overall, non-equidimensional pores were more frequently cuboid-like than plate-like in shape. Clear elongation of pores towards the build direction was infrequent. Instances of notable frequency were due to keyhole instability and melt pool turbulence (high VED or scanning speeds).

The morphology of interconnected porosity was not altered by heat treatment. HIPping effectively reduced porosity in high as-built densification samples by fully closing or partially flattening pores in classes 3-5 with $d_{e q}>50 \mu \mathrm{m}$. A very large irregular pore $\left(d_{e q}=346.45 \mu \mathrm{m}\right)$ was unaffected, thus indicating sensitivity to size. HIPping also appeared to be ineffective at closing very small gas pores, although shape could not be 
measured with certainty. T6 caused pores to reopen and resemble their original shape but densification remained superior to the as-built condition.

Future work will focus on the microstructural behaviour of LPBF-fabricated AlSi10Mg under tension. The influence of different size and shape of pores on tensile properties will be examined, as well as how pore morphology changes at incremental stages of loading by in-situ X $\mu \mathrm{CT}$ scanning. The approach outlined in this paper can be used to study the influence of other processing parameters not investigated here for brevity and can equally be applied to study other LPBF alloys.

\section{Acknowledgements}

The authors gratefully acknowledge the support provided by the EPSRC (grant EP/R021694/1). The authors also wish to thank Rosie Bird at the University of Aberdeen for assisting with Avizo.

\section{Appendix A. Sample Processing Parameters}

Table A1. Sample LPBF processing parameters

\begin{tabular}{|c|c|c|c|}
\hline $\begin{array}{l}\text { Laser Power, } \\
P(\mathrm{~W})\end{array}$ & $\begin{array}{l}\text { Scan Speed, } \\
v(\mathbf{m m} / \mathbf{s})\end{array}$ & $\begin{array}{l}\text { Scan Spacing, } \\
h(\mu \mathrm{m})\end{array}$ & $\begin{array}{l}\text { Layer Thickness, } \\
t(\mu \mathrm{m})\end{array}$ \\
\hline 150 & 500 & 45 & 30 \\
\hline 150 & 500 & 45 & 60 \\
\hline 150 & 500 & 105 & 30 \\
\hline 150 & 500 & 105 & 60 \\
\hline 150 & 1500 & 75 & 30 \\
\hline 150 & 1500 & 75 & 60 \\
\hline 150 & 2500 & 45 & 30 \\
\hline 150 & 2500 & 45 & 60 \\
\hline 150 & 2500 & 105 & 30 \\
\hline 150 & 2500 & 105 & 60 \\
\hline 250 & 500 & 75 & 30 \\
\hline 250 & 500 & 75 & 60 \\
\hline 250 & 1500 & 45 & 30 \\
\hline 250 & 1500 & 45 & 60 \\
\hline $250 *$ & $1500^{*}$ & $75^{*}$ & $30^{*}$ \\
\hline $250 *$ & $1500^{*}$ & $75^{*}$ & $60 *$ \\
\hline 250 & 1500 & 105 & 30 \\
\hline 250 & 1500 & 105 & 60 \\
\hline 250 & 2500 & 75 & 30 \\
\hline 250 & 2500 & 75 & 60 \\
\hline 350 & 500 & 45 & 30 \\
\hline 350 & 500 & 45 & 60 \\
\hline 350 & 500 & 105 & 30 \\
\hline 350 & 500 & 105 & 60 \\
\hline 350 & 1500 & 75 & 30 \\
\hline 350 & 1500 & 75 & 60 \\
\hline 350 & 2500 & 45 & 30 \\
\hline 350 & 2500 & 45 & 60 \\
\hline 350 & 2500 & 105 & 30 \\
\hline 350 & 2500 & 105 & 60 \\
\hline
\end{tabular}




\section{Appendix B. Avizo Results}

Table A2. As-built sample $\mathrm{X} \mu \mathrm{CT}$ results

\begin{tabular}{|c|c|c|c|c|c|c|}
\hline $\begin{array}{l}\text { Sample } P-v-h-t \\
(\mathrm{~W}-\mathrm{mm} / \mathrm{s}-\mu \mathrm{m}-\mu \mathrm{m})\end{array}$ & $\begin{array}{l}V E D \\
\left(\mathrm{~J} / \mathrm{mm}^{3}\right)\end{array}$ & $\begin{array}{l}\text { Porosity } \\
(\%)\end{array}$ & $\begin{array}{l}\text { Scanning } \\
\text { Artefacts }(\%)\end{array}$ & $\begin{array}{l}\text { Densification } \\
(\%)\end{array}$ & $\begin{array}{l}\text { No. of pores } \\
(>1 \text { px })\end{array}$ & $\begin{array}{l}\text { Max. pore } \\
d_{\text {eq }}(\mu \mathrm{m})\end{array}$ \\
\hline $150-500-45-30$ & 222.22 & 0.21 & 0.010 & 99.78 & 1176 & 171.91 \\
\hline $150-500-105-30$ & 95.24 & 0.23 & 0.004 & 99.77 & 1937 & 199.83 \\
\hline $250-1500-75-60 *$ & $37.04 *$ & 0.65 & 0.009 & 99.34 & 5623 & 130.76 \\
\hline $250-1500-75-60 *$ & $37.04 *$ & 0.66 & 0.010 & 99.33 & 5451 & 118.46 \\
\hline $350-500-45-60$ & 259.26 & 0.71 & $<0.001$ & 99.29 & 10319 & 137.2 \\
\hline $250-1500-105-30$ & 52.91 & 0.72 & 0.006 & 99.28 & 2789 & 119.91 \\
\hline $250-2500-75-30$ & 44.44 & 0.80 & 0.007 & 99.190 & 4060 & 111.42 \\
\hline $250-1500-75-60 *$ & $37.04 *$ & 0.81 & 0.003 & 99.186 & 6672 & 157.83 \\
\hline $250-1500-75-30^{+}$ & $74.07^{+}$ & 0.89 & 0.002 & 99.10 & 2387 & 141.19 \\
\hline $350-2500-105-30$ & 44.44 & 0.93 & 0.003 & 99.07 & 6971 & 134.76 \\
\hline $250-1500-75-30^{+}$ & $74.07^{+}$ & 0.96 & 0.005 & 99.03 & 2758 & 143.61 \\
\hline $350-2500-45-60$ & 51.85 & 1.02 & 0.002 & 98.98 & 9786 & 136.91 \\
\hline $350-500-45-30$ & 518.52 & 1.04 & 0.002 & 98.96 & 7570 & 234.62 \\
\hline $250-1500-105-60$ & 26.46 & 1.10 & 0.006 & 98.90 & 4385 & 238.08 \\
\hline $350-2500-45-30$ & 103.70 & 1.12 & $<0.001$ & 98.88 & 5178 & 164.29 \\
\hline $150-500-45-60$ & 111.11 & 1.13 & 0.005 & 98.87 & 1488 & 295.09 \\
\hline $250-1500-45-30$ & 123.46 & 1.19 & 0.003 & 98.81 & 2446 & 144.42 \\
\hline $250-1500-75-30^{+}$ & $74.07^{+}$ & 1.19 & 0.011 & 98.79 & 3414 & 159.08 \\
\hline $150-500-105-60$ & 47.62 & 1.23 & 0.011 & 98.761 & 1684 & 325.27 \\
\hline $250-1500-45-60$ & 61.73 & 1.24 & 0.001 & 98.758 & 7679 & 154.47 \\
\hline $350-2500-105-60$ & 22.22 & 1.45 & 0.025 & 98.52 & 11389 & 237.45 \\
\hline $350-1500-75-30$ & 103.70 & 1.91 & 0.004 & 98.08 & 8802 & 136.31 \\
\hline $350-1500-75-60$ & 51.85 & 2.37 & 0.003 & 97.63 & 21210 & 216.25 \\
\hline $150-1500-75-30$ & 44.44 & 2.90 & 0.015 & 97.08 & 4807 & 446.32 \\
\hline $350-500-105-30$ & 222.22 & 3.25 & 0.004 & 96.74 & 13539 & 239.21 \\
\hline $250-500-75-30$ & 222.22 & 3.39 & 0.001 & 96.61 & 3534 & 170.24 \\
\hline $250-2500-75-60$ & 22.22 & 3.45 & 0.008 & 96.54 & 6113 & 318.5 \\
\hline $250-500-75-60$ & 111.11 & 4.16 & $<0.001$ & 95.84 & 11178 & 169.32 \\
\hline $350-500-105-60$ & 111.11 & 4.44 & 0.001 & 95.56 & 20679 & 201.13 \\
\hline $150-2500-45-30$ & 44.44 & 5.68 & 0.014 & 94.31 & $\{3207\}$ & $\{630.04\}$ \\
\hline $150-1500-75-60$ & 22.22 & 10.21 & 0.006 & 89.78 & $\{3352\}$ & $\{1019.13\}$ \\
\hline $150-2500-45-60$ & 22.22 & 11.59 & 0.035 & 88.37 & $\{2638\}$ & $\{1072.41\}$ \\
\hline $150-2500-105-30$ & 19.05 & 16.08 & 0.014 & 83.91 & $\{3570\}$ & $\{1197.74\}$ \\
\hline $150-2500-105-60$ & 9.52 & 31.35 & 0.019 & 68.64 & $\{5328\}$ & $\{1497.44\}$ \\
\hline
\end{tabular}

\{\} - Porosity interconnected through sub-volume $(2.96 \mu \mathrm{m}$ pixel size $)$

Table A3. Treated sample X $\mu \mathrm{CT}$ results

\begin{tabular}{|c|c|c|c|c|c|c|}
\hline $\begin{array}{l}\text { Sample } P-v-h-t \\
(\mathrm{~W}-\mathrm{mm} / \mathrm{s}-\mu \mathrm{m}-\mu \mathrm{m})\end{array}$ & Condition & $\begin{array}{l}\text { Porosity } \\
(\%)\end{array}$ & $\begin{array}{l}\text { Scanning } \\
\text { Artefacts (\%) }\end{array}$ & $\begin{array}{l}\text { Densification } \\
(\%)\end{array}$ & $\begin{array}{l}\text { No. of pores } \\
(>1 \text { px })\end{array}$ & $\begin{array}{l}\text { Max. pore } \\
d_{\mathrm{eq}}(\mu \mathrm{m})\end{array}$ \\
\hline \multirow[t]{3}{*}{$150-500-105-30$} & As-built & 0.23 & 0.004 & 99.77 & 1937 & 199.83 \\
\hline & HIPped & $<0.01$ & 0.013 & $>99.98$ & 107 & 48.66 \\
\hline & HIPped + T6'd & 0.02 & 0.011 & 99.97 & 1083 & 67.97 \\
\hline \multirow[t]{3}{*}{$250-1500-75-30 *$} & As-built & 0.89 & 0.002 & 99.10 & 2387 & 141.19 \\
\hline & HIPped & $<0.01$ & 0.009 & $>99.98$ & 2082 & 24.15 \\
\hline & HIPped + T6'd & 0.09 & 0.003 & 99.91 & 1173 & 50.38 \\
\hline \multirow[t]{3}{*}{$250-1500-75-30 *$} & As-built & 1.19 & 0.011 & 98.79 & 3414 & 159.08 \\
\hline & HIPped & $<0.01$ & 0.014 & $>99.97$ & 1392 & 77.51 \\
\hline & HIPped + T6’d & 0.07 & 0.023 & 99.91 & 1209 & 66.05 \\
\hline \multirow[t]{2}{*}{$150-500-45-30$} & As-built & 0.21 & 0.010 & 99.78 & 1176 & 171.91 \\
\hline & HIPped & 0.03 & 0.030 & 99.94 & 374 & 140.82 \\
\hline
\end{tabular}




\begin{tabular}{|c|c|c|c|c|c|c|}
\hline & HIPped + T6’d & 0.10 & 0.033 & 99.87 & 426 & 210.76 \\
\hline \multirow[t]{3}{*}{$150-2500-105-30$} & As-built & 16.08 & 0.014 & 83.91 & $\{3570\}$ & $\{1197.74\}$ \\
\hline & HIPped & 15.26 & 0.022 & 84.72 & $\{1024\}$ & $\{1177.72\}$ \\
\hline & HIPped + T6'd & 15.91 & 0.030 & 84.06 & $\{1264\}$ & $\{1194.07\}$ \\
\hline \multirow[t]{3}{*}{$150-2500-105-60$} & As-built & 31.35 & 0.019 & 68.64 & $\{5328\}$ & $\{1497.44\}$ \\
\hline & HIPped & 35.98 & 0.031 & 63.99 & $\{594\}$ & $\{1568.57\}$ \\
\hline & HIPped + T6'd & 30.83 & 0.032 & 69.14 & $\{992\}$ & $\{1489.92\}$ \\
\hline
\end{tabular}

\section{References}

[1] Louvis, E., Fox, P. and Sutcliffe, C.J. (2011). Selective laser melting of aluminium components. Journal of Materials Processing Technology, 211(2), pp.275-284.

[2] Thijs, L., Kempen, K., Kruth, J.-P. and Van Humbeeck, J. (2013). Fine-structured aluminium products with controllable texture by selective laser melting of pre-alloyed AlSi10Mg powder. Acta Materialia, 61(5), pp.1809-1819.

[3] Aboulkhair, N.T., Everitt, N.M., Ashcroft, I. and Tuck, C. (2014). Reducing porosity in AlSi10Mg parts processed by selective laser melting. Additive Manufacturing, 1-4, pp.77-86.

[4] Kempen, K., Thijs, L., Van Humbeeck, J. and Kruth, J.-P. (2015). Processing AlSi10Mg by selective laser melting: parameter optimisation and material characterisation. Materials Science and Technology, 31(8), pp.917-923.

[5] Read, N., Wang, W., Essa, K. and Attallah, M.M. (2015). Selective laser melting of AlSi10Mg alloy: Process optimisation and mechanical properties development. Materials \& Design, 65, pp.417-424.

[6] Aboulkhair, N.T., Maskery, I., Tuck, C., Ashcroft, I. and Everitt, N.M. (2016). The microstructure and mechanical properties of selectively laser melted AlSi10Mg: The effect of a conventional T6-like heat treatment. Materials Science and Engineering: A, 667, pp.139-146.

[7] Takata, N., Kodaira, H., Sekizawa, K., Suzuki, A. and Kobashi, M. (2017). Change in microstructure of selectively laser melted AlSi10Mg alloy with heat treatments. Materials Science and Engineering: A, 704, pp.218-228.

[8] Wei, P., Wei, Z., Chen, Z., Du, J., He, Y., Li, J. and Zhou, Y. (2017). The AlSi10Mg samples produced by selective laser melting: single track, densification, microstructure and mechanical behavior. Applied Surface Science, 408, pp.38-50.

[9] Rosenthal, I., Shneck, R. and Stern, A. (2018). Heat treatment effect on the mechanical properties and fracture mechanism in AlSi10Mg fabricated by additive manufacturing selective laser melting process. Materials Science and Engineering: A, 729, pp.310-322.

[10] Wang, L.F., Sun, J., Yu, X.L., Shi, Y., Zhu, X.G., Cheng, L.Y., Liang, H.H., Yan, B. and Guo, L.J. (2018). Enhancement in mechanical properties of selectively laser-melted AlSi10Mg aluminum alloys by T6-like heat treatment. Materials Science and Engineering: A, 734, pp.299-310. 
[11] Zhuo, L., Wang, Z., Zhang, H., Yin, E., Wang, Y., Xu, T. and Li, C. (2019). Effect of post-process heat treatment on microstructure and properties of selective laser melted AlSi10Mg alloy. Materials Letters, 234, pp.196-200.

[12] Girelli, L., Tocci, M., Gelfi, M. and Pola, A. (2019). Study of heat treatment parameters for additively manufactured AlSi10Mg in comparison with corresponding cast alloy. Materials Science and Engineering: A, 739, pp.317-328.

[13] Cai, X., Malcolm, A.A., Wong, B.S. and Fan, Z. (2015). Measurement and characterization of porosity in aluminium selective laser melting parts using X-ray CT. Virtual and Physical Prototyping, 10(4), pp.195206.

[14] Qiu, C., Yue, S., Adkins, N.J.E., Ward, M., Hassanin, H., Lee, P.D., Withers, P.J. and Attallah, M.M. (2015). Influence of processing conditions on strut structure and compressive properties of cellular lattice structures fabricated by selective laser melting. Materials Science and Engineering, 628, pp.188-197.

[15] Maskery, I., Aboulkhair, N.T., Corfield, M.R., Tuck, C., Clare, A.T., Leach, R.K., Wildman, R.D., Ashcroft, I.A. and Hague, R.J.M. (2016). Quantification and characterisation of porosity in selectively laser melted Al-Si10-Mg using X-ray computed tomography. Materials Characterization, 111, pp.193-204.

[16] Tradowsky, U., White, J., Ward, R.M., Read, N., Reimers, W. and Attallah, M.M. (2016). Selective laser melting of AlSi10Mg: Influence of post-processing on the microstructural and tensile properties development. Materials \& Design, 105, pp.212-222.

[17] Larrosa, N.O., Wang, W., Read, N., Loretto, M.H., Evans, C., Carr, J., Tradowsky, U., Attallah, M.M. and Withers, P.J. (2018). Linking microstructure and processing defects to mechanical properties of selectively laser melted AlSi10Mg alloy. Theoretical and Applied Fracture Mechanics, 98, pp.123-133.

[18] Romano, S., Abel, A., Gumpinger, J., Brandão, A.D. and Beretta, S. (2019). Quality control of AlSi10Mg produced by SLM: Metallography versus CT scans for critical defect size assessment. Additive Manufacturing, 28, pp.394-405.

[19] Kan, W.H., Nadot, Y., Foley, M., Ridosz, L., Proust, G. and Cairney, J.M. (2019). Factors that affect the properties of additively-manufactured AlSi10Mg: Porosity versus microstructure. Additive Manufacturing, 29, p. 100805 .

[20] DebRoy, T., Wei, H.L., Zuback, J.S., Mukherjee, T., Elmer, J.W., Milewski, J.O., Beese, A.M., WilsonHeid, A., De, A. and Zhang, W. (2018). Additive manufacturing of metallic components - Process, structure and properties. Progress in Materials Science, 92, pp.112-224.

[21] Olakanmi, E.O. (2013). Selective laser sintering/melting (SLS/SLM) of pure Al, Al-Mg, and Al-Si powders: Effect of processing conditions and powder properties. Journal of Materials Processing Technology, 213(8), pp.1387-1405.

[22] Qiu, C., Panwisawas, C., Ward, M., Basoalto, H.C., Brooks, J.W. and Attallah, M.M. (2015). On the role of melt flow into the surface structure and porosity development during selective laser melting. Acta Materialia, 96, pp.72-79. 
[23] Sercombe, T.B. and Li X. (2016). Selective laser melting of aluminium and aluminium metal matrix composites: review. Materials Technology, 31(2), pp.77-85.

[24] Kartal, M.E., Dugdale, L.H., Harrigan, J.J., Siddiq, M.A., Pokrajac, D. and Mulvihill, D.M. (2017). Three-dimensional in situ observations of compressive damage mechanisms in syntactic foam using X-ray microcomputed tomography. Journal of Materials Science, 52(17), pp.10186-10197.

[25] Georgilas K., Khan R.H.U. and Kartal M.E. (2020). The influence of pulsed laser powder bed fusion process parameters on Inconel 718 material properties. Materials Science and Engineering A. 769, 138527.

[26] Ridler, T.W. and Calvard, S. (1978). Picture thresholding using an iterative selection method. IEEE Transactions on Systems, Man, and Cybernetics, 8(8), pp.630-632.

[27] Blott, S.J. and Pye, K. (2008). Particle shape: a review and new methods of characterization and classification. Sedimentology, 55, pp.31-63.

[28] Soete, J., Kleipool, L.M., Claes, H., Claes, S., Hamaekers, H., Kele, S., Özkul, M., Foubert, A., Reijmer, J.J.G. and Swennen, R. (2015). Acoustic properties in travertines and their relation to porosity and pore types. Marine and Petroleum Geology, 59, pp.320-335.

[29] Schmitt, M., Halisch, M., Müller, C. and Fernandes, C.P. (2016). Classification and quantification of pore shapes in sandstone reservoir rocks with 3-D X-ray micro-computed tomography. Solid Earth, 7(1), pp.285-300.

[30] Wang, L.-Z., Wang, S. and Wu, J.-J. (2017). Experimental investigation on densification behavior and surface roughness of AlSi10Mg powders produced by selective laser melting. Optics \& Laser Technology, 96, pp.88-96.

[31] Trapp, J., Rubenchik, A.M., Guss, G. and Matthews, M.J. (2017). In situ absorptivity measurements of metallic powders during laser powder-bed fusion additive manufacturing. Applied Materials Today, 9, pp.341-349.

[32] Shao, S., Mahtabi, M.J., Shamsaei, N. and Thompson, S.M. (2017). Solubility of argon in laser additive manufactured $\alpha$-titanium under hot isostatic pressing condition. Computational Materials Science, 131, pp.209-219. 


\section{Highlights}

Manuscript title: Classifying shape of internal pores within AlSi10Mg alloy manufactured by laser powder bed fusion using 3D X-ray micro computed tomography: influence of processing parameters and heat treatment

- AlSi10Mg samples were manufactured by laser powder bed fusion

- Very low to high densifications were produced using a range of process parameters

- Pores were analysed by X-ray micro computed tomography and classified by shape

- Pores were fully or partially closed (flattened) by HIPping

- T6 after HIPping caused pores to reopen and resemble their original shape 